\title{
The Energy Complexity of BFS in Radio Networks
}

\author{
Yi-Jun Chang \\ ETH Zürich
}

\author{
Varsha Dani \\ Univ. of New Mexico
}

\author{
Thomas P. Hayes* \\ Univ. of New Mexico
}

\author{
Seth Pettie $^{\dagger}$ \\ Univ. of Michigan
}

\begin{abstract}
We consider a model of energy complexity in Radio Networks in which transmitting or listening on the channel costs one unit of energy and computation is free. This simplified model captures key aspects of battery-powered sensors: that battery-life is most influenced by transceiver usage, and that at low transmission powers, the actual cost of transmitting and listening are very similar.

The energy complexity of tasks in single-hop (clique) networks are well understood [11, 35. 6. 22. . Recent work of Chang et al. [10] considered energy complexity in multi-hop networks and showed that Broadcast admits an energy-efficient protocol, by which we mean each of the $n$ nodes in the network spends $O(\operatorname{polylog}(n))$ energy. This work left open the strange possibility that all natural problems in multi-hop networks might admit such an energy-efficient solution.

In this paper we prove that the landscape of energy complexity is rich enough to support a multitude of problem complexities. Whereas Broadcast can be solved by an energy-efficient protocol, exact computation of Diameter cannot, requiring $\Omega(n)$ energy. Our main result is that BreadthFirstSearch has sub-polynomial energy complexity at most $2^{O(\sqrt{\log n \log \log n})}=n^{o(1)}$; whether it admits an efficient $O(\operatorname{polylog}(n))$-energy protocol is an open problem.

Our main algorithm involves recursively solving a generalized BFS problem on a "cluster graph" introduced by Miller, Peng, and Xu [34]. In this application, we make crucial use of a close relationship between distances in this cluster graph, and distances in the original network. This relationship is new and may be of independent interest.

We also consider the problem of approximating the network Diameter. From our main result, it is immediate that Diameter can be 2-approximated using $n^{o(1)}$ energy per node. We observe that, for all $\epsilon>0$, approximating Diameter to within a $(2-\epsilon)$ factor requires $\Omega(n)$ energy per node. However, this lower bound is only due to graphs of very small diameter; for large-diameter graphs, we prove that the diameter can be nearly 3/2-approximated using $O\left(n^{1 / 2+o(1)}\right)$ energy per node.
\end{abstract}

\footnotetext{
*Supported by NSF CAREER award CCF-1150281.

${ }^{\dagger}$ Supported by NSF grants CCF-1514383, CCF-1637546, and CCF-1815316.
} 


\section{Introduction}

Consider a network of $n$ tiny sensors scattered throughout a National Park. We'd like the sensors to organize themselves, so that in the event of a forest fire, say, information about it can be efficiently broadcast to the entire network.

In this extremely low power setting, sensors would need to spend most of their time with their transceiver units shut off to conserve power. In a steady state, we might expect that we have a good labelling of the nodes, and each node with label $i$ wakes up at times of the form $j P+i$, where $j$ runs through every positive integer, and $P$, the polling period, is also a positive integer. Each node wakes up just long enough to receive a message and forward it on any neighbors with label $i+1$. In this way, at the expense of adding $P$ to the latency, the nodes are able to reduce their power consumption by a factor of $P$, compared to the always-on scenario.

Once $P$ has been optimized, which should be a function of the available power, the next issue is how to find a good labelling efficiently. In this paper we focus mainly on the problem of computing BFS labelings: a given source $s$ has label zero, and all other devices label themselves by the distance (in hops) to $s$. Such a labeling gives a 2-approximation to the diameter, and via up-casts and downcasts, allows for time- and energy-efficient dissemination of a message from any origin. Thus, the problem of finding a BFS labelling is a very natural question in this context.

\section{$1.1 \quad$ The Model}

We work within the classic Radio Network model [12, but in contrast to most prior work in this model, we treat energy (defined below) as the primary measure of complexity and time to be important, but secondary.

There are $|V|$ devices associated with the nodes of an unknown undirected graph $G=(V, E)$. Time is partitioned into discrete steps. All devices agree on time zero 1 and agree on some upper bound $n \geq|V|$. In each timestep, each device performs some computation and chooses to either idle, listen to the channel, or transmit a message. If a device $v$ chooses to listen, and exactly one device $u \in N(v)$ transmits a message $m_{u}$, then $v$ receives $m_{u}$. In all other cases, $v$ receives no feedback from the environment 2 Devices can locally generate unbiased random bits; there is no shared randomness. Let RN $[b]$ denote this Radio Network model, where $b$ is the maximum number of bits per message. All of our algorithms work in $\mathrm{RN}[O(\log n)]$ and all our lower bounds apply even to $\mathrm{RN}[\infty]$.

Cost Measures. An algorithm runs in time $t$ if all devices halt and return their output by timestep $t$. Typically the algorithm is randomized, with some probability of failure, but $t$ is a function of $n$ or other given parameters, not a random variable. The energy cost of $v \in V$ is the number of timesteps for which $v$ is listening or transmitting. (This is motivated by the fact that the sleep mode of tiny devices is so efficient that it is reasonable to approximate its energy-cost by zero, and that transceiver usage is often the most expensive part of a computation. Moreover, at low transmission

\footnotetext{
${ }^{1}$ Synchronizing devices in an energy-efficient manner is an interesting open problem. In some situations it makes sense to assume the devices begin in a synchronized state, e.g., if the sensors are simultaneously turned on and dropped from an airplane on the aforementioned National Park.

${ }^{2}$ Here $N(v)=\{u \mid\{u, v\} \in E(G)\}$ is the neighborhood of $v$. A more powerful model allows for collision detection, i.e., differentiation between zero and two or more transmitters in $N(v)$. Since collision detection only gives a polylog $(n)$ advantage in any complexity measure (Local-Broadcast in Section 2 allows each vertex to differentiate between zero and two or more transmitters in polylog $(n)$ rounds w.h.p.) and we are insensitive to such factors, we assume the weakest model, without collision detection.
} 
powers, transmitting and listening are comparable; see, e.g., [37, Fig. 2] and [5, Table 1].) The energy cost of the algorithm is the maximum energy cost of any device.

Energy Complexity. Most prior work on energy complexity has focused on single-hop (clique) networks, typically under the assumption that $|V|=n$ is unknown, and that some type of collisiondetection is available 3 Because of the high degree of symmetry, there are only so many interesting problems in single-hop networks. Nakano and Olariu 35, proved that the Initialization problem (assign devices distinct IDs in $\{1, \ldots,|V|=n\}$ ) can be solved with $O(\log \log n)$ energy. Bender et al. [6] showed that with collision-detection, all $n$ devices holding messages can transmit all of them using $O\left(\log \left(\log ^{*} n\right)\right)$ energy. Chang et al. [11 proved that $\Theta\left(\log \left(\log ^{*} n\right)\right)$ is optimal, and more generally, settled the complexity of LeaderElection and ApproximateCounting (estimating " $n$ ") in all the collision-detection models, with and without randomization. It was proved that collision-detection gives two exponential advantages in energy complexity. With randomization, LeaderElection/ApproximateCounting takes $\Theta\left(\log ^{*} n\right)$ energy (without CD) or $\Theta\left(\log \left(\log ^{*} n\right)\right)$ energy (with CD), and deterministically, they take $\Theta(\log N)$ energy (without CD [22]) and $\Theta(\log \log N)$ energy (with CD), where devices initially have IDs in $[N]$. See also [21, 20, 22, 23, 24]. Three-way tradeoffs between time, energy, and error probability were studied by Chang et al. [11] and Kardas et al. [27].

Very recently Chang et al. [10] extended the single-hop notion of energy complexity to multi-hop networks ( $G$ is not a clique), and proved nearly sharp upper and lower bounds on Broadcast, both in $\mathrm{RN}[O(\log n)]$ and the same model when listeners have collision detection. Without CD the energy complexity of Broadcast is between $\Omega\left(\log ^{2} n\right)$ and $O\left(\log ^{3} n\right)$; with CD it is between $\Omega(\log n)$ and $O\left(\frac{\log n \log \log n}{\log \log \log n}\right)$.

Other Energy Models. Other notions of energy complexity have been studied in radio networks. For example, when distances between devices are very large, transmitting is significantly more expensive than listening, and it makes sense to design algorithms that minimize the worst-case number of transmissions per device. Gasnieniec et al. [15], Klonowski and Pajak [29], and Berenbrink et al. [7] studied broadcast and gossiping problems under this cost model. Klonowski and Sulkowska [30] defined a distributed model in which devices are scattered randomly at points in $\left[n^{1 / d}\right]^{d}$ and can choose their transmission power dynamically. Several works have looked at energy complexity against an adversarial jammer, where the energy cost is sometimes a function of the adversary's energy budget. See, e.g., [32, 25, 17, 28].

Time Complexity. Most prior work in the RN model has studied the time complexity of basic primitives such as LeaderElection, Broadcast, BFS, etc. We review a few results most relevant to our work. Bar-Yehuda et al.'s [3] decay algorithm solves BFS in $O\left(D \log ^{2} n\right)$ time and Broadcast in $O\left(D \log n+\log ^{2} n\right)$ time. Here $D$ is the diameter of the network. Since $\Omega(D)$ is an obvious lower bound, the question is which log-factors are necessary. Alon et al. [2] proved that the additive $\log ^{2} n$ term is necessary in a strong sense: even with full knowledge of the graph topology, Broadcast needs $\Omega\left(\log ^{2} n\right)$ time even when $D=O(1)$. Kushilevitz and Mansour [31] proved that if devices are forbidden from transmitting before hearing the message, then $\Omega(D \log (n / D))$ time in necessary. Czumaj and Davies [14] (improving [18) gave a Broadcast algorithm running in $O\left(D \log _{D} n+\right.$ polylog $(n))$ time, which is optimal when $D>n^{\epsilon}$. These Broadcast algorithms do not solve BFS.

\footnotetext{
${ }^{3}$ Sender-side CD enables devices to detect if another device is transmitting; receiver-side CD lets receivers detect if at least two devices are transmitting.
} 
Improving the classic $O\left(D \log ^{2} n\right)$ decay algorithm for BFS, Ghaffari and Haeupler [16] solve BFS in $O(D \log (n) \log \log (n)+$ polylog $(n))$ time.

New Results. It is useful to coarsely classify energy-efficiency bounds as either feasible or infeasible. We consider polylog $(n)$ energy to be feasible and polynomial energy $n^{\Omega(1)}$ to be infeasible 4 It is not immediately obvious that there are any natural, infeasible problems, especially if we are considering the full power of $\mathrm{RN}[\infty]$, where message congestion is not an issue. In this paper we demonstrate that the energy landscape is rich, and that even coarsely classifying the energy complexity of simple problems is technically challenging and demands the development of new algorithm design techniques. Our results are as follows

- We develop a recursive BreadthFirstSearch algorithm in $\mathrm{RN}[O(\log n)]$ with "intermediate" energy-complexity $2^{O(\sqrt{\log n \log \log n})}=n^{o(1)}$. The algorithm involves simulating itself on a clustered version of the input graph. Due to the nature of the RN model, this simulation is not free, but incurs a polylogarithmic increase in energy, which restricts the profitable depth of recursion to be at most $\sqrt{\log n / \log \log n}$.

- We give examples of some "hard" problems in energy-complexity, even when the model is $\mathrm{RN}[\infty]$. The problem of deciding whether $\operatorname{diam}(G)$ is 1 or at least 2 takes $\Omega(n)$ energy; in this case the hard graph $G$ is dense. We adapt the construction of [1] (designed for the CONGEST model) to show that even on sparse graphs, with arboricity $O(\log n)$, deciding whether $\operatorname{diam}(G)$ is 2 or at least 3 takes $\tilde{\Omega}(n)$ energy.

- To complement the hardness results, we show that Diameter can be nearly $3 / 2$-approximated 5 in $\mathrm{RN}[O(\log n)]$ with $O\left(n^{1 / 2+o(1)}\right)$ energy, by adapting [19, 38] and using our new BreadthFirstSearch routine.

The existence of a subpolynomial-energy BreadthFirstSearch algorithm is somewhat surprising for information-theoretic reasons. Observe that the number of edges in $E(G)$ that are collectively discovered by all devices is at most the number of messages successfully received, which itself is at most the aggregate energy cost. Thus, if the per-device energy cost is $n^{o(1)}$, we can never hope to know about more than $n^{1+o(1)}$ edges in $E(G)$ - a negligible fraction of the input on dense graphs! On the other hand, it is possible to efficiently verify the non-existence of many non-edges. Given a candidate BFS-labeling, for example, it is straightforward to verify its correctness with polylog $(n)$ energy.

Organization. In Section 2] we review the Miller-Peng-Xu [34] clustering algorithm and prove that it preserves distances better than previously known. In Section 3 we define some communications primitives and prove that they can be executed on the cluster graph (as if it were an $\mathrm{RN}[O(\log n)]$ network) at the cost of a polylogarithmic factor increase in energy usage. In Section 4 we design and analyze a recursive BFS algorithm, which uses $2^{O(\sqrt{\log n \log \log n})}$ energy. In Section 5 we consider the energy cost of approximately computing the network's Diameter.

\section{Cluster Partitioning}

Miller, Peng, and $\mathrm{Xu}$ [34] introduced a remarkably simple algorithm for partitioning a given graph into vertex-disjoint clusters with certain desirable properties. In this section we prove that the

\footnotetext{
${ }^{4}$ These definitions seem to be robust to certain modeling assumptions, e.g., whether collision detection is available.

${ }^{5}$ I.e., it returns a value in the range $\left[\left\lfloor\frac{2}{3} \operatorname{diam}(G)\right\rfloor, \operatorname{diam}(G)\right]$.
} 

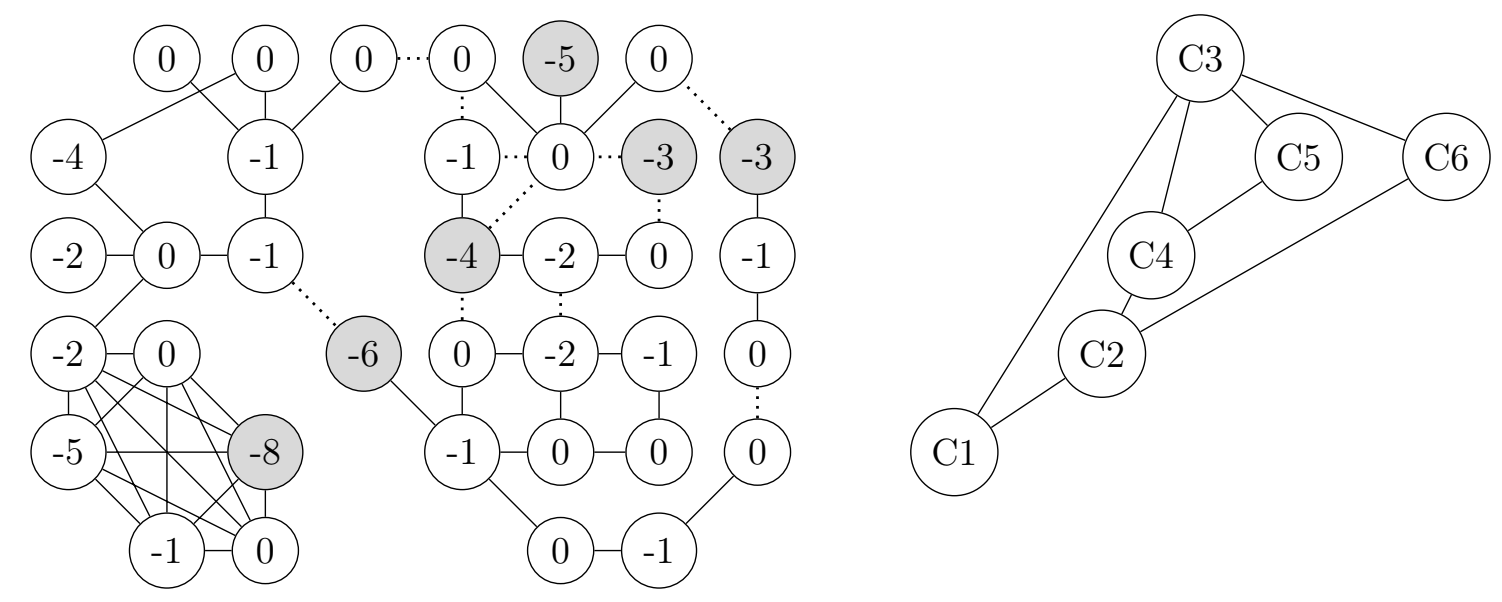

Figure 1: Constructing a cluster graph. At left, the original graph; with the (rounded) start time, $-\delta_{v}$, marked on each vertex. The cluster centers have been darkened, and the dotted lines indicate edges that cross a cluster boundary. At right, the corresponding cluster graph. Note that the distances in the cluster graph are broadly proportional to the original distances, but can vary significantly.

MPX clustering approximately preserves relative distances from the original graph significantly better than previously known.

Given a graph $G=(V, E)$, and a parameter $\beta$, each vertex $v \in V$ independently samples a random variable $\delta_{v} \sim \operatorname{Exponential}(\beta)$ from the exponential distribution with mean $1 / \beta$. Assign each $v$ to the "cluster" centered at $u \in V$ that minimizes $\operatorname{dist}_{G}(v, u)-\delta_{u}$. Equivalently, we may think of a cluster forming at each vertex $u$ at time $-\delta_{u}$, and spreading through the graph at a uniform rate of one edge per time unit. Each vertex $v$ is absorbed into the first cluster to reach it, if this happens prior to time $-\delta_{v}$, when it would start growing its own cluster. Refer to Figure 1 , Throughout the paper, we only choose $\beta$ such that $1 / \beta$ is an integer.

Miller et al. 34] were primarily interested in this construction because the algorithm parallelizes well, the clusters have diameter $O(\log (n) / \beta)$ w.h.p., and a $O(\beta)$-fraction of the edges are "cut," having their endpoints in distinct clusters. Haeupler and Wajc [18] observed that this algorithm can be efficiently implemented in the Radio Network model [12, 13], with only minor modifications.

\subsection{The Cluster Graph as a Distance Proxy}

Define $\mathrm{Cl}(u)$ to be the cluster containing $u$. The cluster graph, $\operatorname{cluster}(G, \beta)=G^{*}=\left(V^{*}, E^{*}\right)$ is defined by

$$
\begin{aligned}
V^{*} & =\{\mathrm{Cl}(u) \mid u \in V(G)\} \\
\text { and } E^{*} & =\{(\mathrm{Cl}(u), \mathrm{Cl}(v)) \mid(u, v) \in E(G), \mathrm{Cl}(u) \neq \mathrm{Cl}(v)\} .
\end{aligned}
$$

To prove that distances in $G^{*}$ are a good proxy for distances in $G$, we make use of the following lemma, which is a slight variant of lemmas by Miller, Peng, Vladu, and Xu [33, Lemma 2.2] and Haeupler and Wajc [18, Corollary 3.8]. We include a proof for completeness.

Define $\operatorname{Ball}_{G}(v, \ell)=\left\{u \in V \mid \operatorname{dist}_{G}(u, v) \leq \ell\right\}$ to be the ball of radius $\ell$ around $v$. 
Lemma 2.1. Let $G^{*}=\operatorname{cluster}(G, \beta)$ be the cluster graph for $G$. For every positive integer $j$ and $\ell>0$, the probability that the number of $G^{*}$-clusters intersecting $\operatorname{Ball}_{G}(v, \ell)$ is more than $j$ is at most

$$
(1-\exp (-2 \ell \beta))^{j}
$$

Proof. Condition on the time $t$ that the $(j+1)$ st signal would reach vertex $v$, as well as on the identities $v_{1}, \ldots, v_{j}$ of the vertices whose signals reach $v$ before time $t$. Due to the memoryless property of the exponential distribution, each of these arrival times are independently distributed as $\min \left\{t, \operatorname{dist}\left(v_{i}, v\right)\right\}-X \leq t-X$, where $X \sim \operatorname{Exponential}(\beta)$.

Now, if $\max _{1 \leq i \leq j} X_{i}>2 \ell$, then $\operatorname{Ball}_{G}(v, \ell)$ cannot intersect any clusters except those centered at $v_{1}, \ldots, v_{j}$, because they do not reach $\operatorname{Ball}_{G}(v, \ell)$ until times $\geq t-\ell$, whereas the first signal reached $v$ before time $t-2 \ell$, and has therefore already flooded all of $\operatorname{Ball}_{G}(v, \ell)$ before time $t-\ell$. Thus,

$\mathbf{P}\left(\operatorname{Ball}_{G}(v, \ell)\right.$ intersects more than $j$ clusters $) \leq \mathbf{P}\left(\forall i \in[1, j], X_{i} \leq 2 \ell\right)=(1-\exp (-2 \ell \beta))^{j}$.

A natural way to show that $G^{*}$ approximately preserves distances in $G$ is to consider the fraction of edges in a shortest path that are "cut" by the partition, which corresponds to applying Lemma 2.1 with $\ell=1 / 2$ and $j=16$ This was the approach taken in [10], but it only guarantees that the fraction of edges cut concentrates around its expectation $(O(\beta))$ for paths of length $\tilde{\Omega}\left(\operatorname{poly}\left(\beta^{-1}\right)\right)$. In Lemmas 2.2 and 2.3 we use Lemma 2.1 in a different way to bound the ratio of distances in $G$ to those in $G^{*}$, which works even for relatively short distances. Lemma 2.2 applies to all distances (and suffices for our BFS application in Section (4) whereas Lemma 2.3 applies to distances $\Omega\left(\beta^{-1} \log ^{2} n\right)$.

Lemma 2.2. Let $G^{*}=\operatorname{cluster}(G, \beta)$ be a clustering of $G$. There exists a constant $C$ such that for every pair $u, v \in V(G)$,

$$
\mathbf{P}\left(\operatorname{dist}_{G^{*}}(\mathrm{Cl}(u), \mathrm{Cl}(v)) \in\left[\left\lfloor\frac{\operatorname{dist}_{G}(u, v) \cdot \beta}{8 \log (n)}\right\rfloor,\left\lceil\operatorname{dist}_{G}(u, v) \cdot \beta\right\rceil \cdot C \log (n)\right]\right) \geq 1-\frac{1}{n^{3}} .
$$

More generally, let $P=(u, \ldots, v)$ be any length-d path connecting $u$ and $v$. With probability $1-\frac{1}{n^{3}}$, there exists a path $P^{*}$ in $G^{*}$ connecting $\mathrm{Cl}(u)$ and $\mathrm{Cl}(v)$ with length at most $d \cdot C \beta \log (n)$, where each cluster in $P^{*}$ intersects $P$.

Proof. First observe that the probability of any $\delta_{v}$-value being outside $[0,4 \log (n) / \beta)$ is $\ll n^{-4}$ and hence all clusters have radius less than $4 \log (n) / \beta$ with probability $\ll n^{-3}$. This gives the lower bound on $\operatorname{dist}_{G^{*}}(u, v)$.

For the upper bound, define $\ell$ to be the integer $1 / \beta$. Fix any length- $d$ path $P$ from $u$ to $v$ (e.g., a shortest path, with $\left.d=\operatorname{dist}_{G}(u, v)\right)$, and cover its vertices with $\left\lceil\frac{d}{2 \ell+1}\right\rceil$ paths of length $2 \ell$. Applying Lemma 2.1 to the center vertex $u^{\prime}$ of one of these subpaths, we conclude that the number of clusters that intersect $\operatorname{Ball}_{G}\left(u^{\prime}, \ell\right)$, (which includes the entire subpath) is more than $j$ with probability

$$
(1-\exp (-2 \beta \ell))^{j}=(1-\exp (-2))^{j}
$$

Choosing $j$ to be the appropriate multiple of $\log (n)$, we can make this probability $\ll n^{-4}$. Taking a union bound over the $\approx \beta d / 2<n$ subpaths, the probability that any subpath intersects more than $C \log (n)$ clusters is $\ll n^{-3}$. This concludes the proof.

\footnotetext{
${ }^{6}$ One imagines a vertex $v_{e}$ in the middle of an edge $e ; e$ is cut iff Ball ${ }_{G}\left(v_{e}, 1 / 2\right)$ intersects two clusters, which must cover distinct endpoints of $e$.
} 
Lemma 2.2 suffices to achieve our main result, BFS labeling in $2^{O(\sqrt{\log n \log \log n})}$ energy, but the exponent can be improved by a constant factor by using Lemma 2.3 whenever applicable. We include the proof of Lemma 2.3 since it may be of independent interest.

Lemma 2.3. Let $G^{*}=\operatorname{cluster}(G, \beta)$ be a clustering of $G$. There exists a constant $C$ such that for every pair $u, v \in V(G)$

$$
\mathbf{P}\left(\operatorname{dist}_{G^{*}}(\mathrm{Cl}(u), \mathrm{Cl}(v)) \in\left[\frac{\operatorname{dist}_{G}(u, v) \cdot \beta}{8 \log (n)}, \operatorname{dist}_{G}(u, v) \cdot C \beta\right]\right) \geq 1-\frac{1}{n^{3}} .
$$

Proof. We condition on the event that all cluster radii are at most $4 \log (n) / \beta$, which fails to hold with probability $\ll n^{-3}$. As before, the lower bound on $\operatorname{dist}_{G^{*}}(u, v)$ follows from this event. Furthermore, this implies that sufficiently distant segments on the shortest $u-v$ path are essentially independent.

As before, cover the vertices of the shortest $u-v$ path with length- $2 \ell$ subpaths, $\ell=1 / \beta$, and color the subpaths with $4 \log (n)+1$ colors such that any two subpaths of the same color are at distance at least $8 \log (n) / \beta$. Each color-class contains $\Omega(\log n)$ subpaths. By Lemma 2.1 and (1), the number of clusters intersecting subpaths of a particular color class is stochastically dominated by the sum of $\Omega(\log n)$ geometrically distributed random variables with constant expectation $\frac{1}{1-(1-\exp (-2))}=$ $\exp (2)$. By a Chernoff bound, the probability that this sum deviates from its expectation by more than a constant factor is $1 / \operatorname{poly}(n)$. Hence, for sufficiently large $C$ (controlling the number of summands and the tolerable deviation) the probability that any color-class hits too many distinct clusters is $\ll n^{-3}$.

Remark 2.1. Lemma 2.3 cannot be improved by more than constant factors. It is easy to construct families of graphs for which both the upper and lower bounds are tight, with high probability, depending on which vertex pairs are chosen.

\subsection{Distributed Implementation}

The definition of cluster $(G, \beta)$ immediately lends itself to a distributed implementation in radio networks, as was noted in [18]. For completeness we show how it can be reduced to calls to Local-Broadcast.

Local-Broadcast: We are given two disjoint vertex sets $\mathcal{S}$ and $\mathcal{R}$, where each vertex $u \in \mathcal{S}$ holds a message $m_{u}$. An Local-Broadcast algorithm guarantees that for every $v \in \mathcal{R}$ with $N(v) \cap \mathcal{S} \neq \emptyset$, with probability $1-f, v$ receives some message $m_{u}$ from at least one vertex $u \in N(v) \cap \mathcal{S}$. We only apply this routine with $f=1 / \operatorname{poly}(n)$.

Lemma 2.4. Local-Broadcast can be implemented in $O\left(\log \Delta \log f^{-1}\right)$ time and energy, where $\Delta \leq$ $n-1$ is an upper bound on the maximum degree. Senders use $O\left(\log f^{-1}\right)$ energy; receivers that hear a message use $O(\log \Delta)$ energy in expectation; receivers that hear no message use $O\left(\log \Delta \log f^{-1}\right)$ energy.

Proof. This lemma follows from a small modification to the Decay algorithm [4, which is known to be optimal in terms of time; see Newport [36]. For the sake of completeness, we provide a proof here. Each sender $u \in \mathcal{S}$ repeats the following $O\left(\log f^{-1}\right)$ times. Randomly pick an $X_{u} \in[1, \log \Delta]$ such that $\mathbf{P}\left(X_{u}=t\right) \geq 2^{-t}$ and transmit $m_{u}$ at time step $X_{u}$. The energy of any sender is clearly $O\left(\log f^{-1}\right)$ with probability 1 . For a receiver $v \in \mathcal{R}$, if the number of senders in $N(v)$ is in the range $\left[2^{t-1}, 2^{t}\right], v$ will receive some message with constant probability in the th timestep of every iteration. Receivers with no adjacent sender will never detect this, and spend $\Theta\left(\log \Delta \log f^{-1}\right)$ energy. 
We show that cluster $(G, \beta)$ can be computed, w.h.p., using $4 \log (n) / \beta$ Local-Broadcasts in the communication network $G=(V, E)$. Every vertex $u$ will learn its cluster-identifier $\operatorname{ID}(\mathrm{Cl}(u)))$ and get a label $\mathcal{L}(v)$ such that $\mathcal{L}(v)=0$ iff $v$ is a cluster center and $\mathcal{L}(v)=i$ iff there is a $u \in N(v)$ with $\mathcal{L}(u)=i-1$ such that $\mathrm{Cl}(u)=\mathrm{Cl}(v)$. If $\mathcal{L}(v)=i$, we say that $v$ is at layer $i$.

The graph $\operatorname{cluster}(G, \beta)$ is constructed as follows. Every vertex $v$ picks a value $\delta_{v} \sim$ Exponential $(\beta)$ and sets its start time to be $\operatorname{start}_{v} \leftarrow\left\lceil\frac{4 \log (n)}{\beta}-\delta_{v}\right\rceil$. With probability at least $1-1 / n^{3}$, all start times are positive. For $i=1$ to $4 \log (n) / \beta$, do the following. At the beginning of the $i$ th iteration, if $v$ is not yet in any cluster and $\operatorname{start}_{v}=i$, then $v$ becomes a cluster center and sets $\mathcal{L}(v)=0$. During the $i$ th iteration, we execute Local-Broadcast with $\mathcal{S}$ being the set of all clustered vertices and $\mathcal{R}$ the set of all as-yet unclustered vertices. The message of $u \in \mathcal{S}$ contains $\operatorname{ID}(\mathrm{Cl}(u))$ and $\mathcal{L}(u)$. Any vertex $v \in \mathcal{R}$ receiving a message from $u \in \mathcal{S}$ joins $u$ 's cluster and sets $\mathcal{L}(v)=\mathcal{L}(u)+1$. Lemma 2.5 follows immediately from the above construction.

Lemma 2.5. The cluster graph cluster $(G, \beta)$ can be constructed using $4 \log (n) / \beta$ Local-Broadcasts with probability $1-1 / n^{3}$. This takes $O\left(\log ^{3}(n) / \beta\right)$ time and $O\left(\log ^{3}(n) / \beta\right)$ energy per vertex.

\section{Communication Primitives for the Cluster Graph}

Our BFS algorithm forms a cluster graph $G^{*}$ and computes BFS recursively on numerous subgraphs of $G^{*}$. In order for this type of recursion to work, we need to argue that algorithms on the (abstract) $G^{*}$ can be simulated, with some time and energy cost, on the underlying $G$. We focus on algorithms that are composed exclusively of calls to Local-Broadcast (as our BFS algorithm is), but the method can be used to simulate arbitrary radio network algorithms.

We use the primitives Down-cast and Up-cast to allow cluster centers to disseminate information to their constituents and gather information from some constituent.

Down-cast: There is a set $\mathcal{U}$ of vertices such that each $u \in \mathcal{U}$ is a cluster center, and the goal is to let each $u \in \mathcal{U}$ broadcast a message $m_{u}$ to all members of $\mathrm{Cl}(u)$.

Up-cast: There is a set $\mathcal{U}$ of vertices such that each $u \in \mathcal{U}$ wants to deliver a message $m_{u}$ to the center of $\mathrm{Cl}(u)$. Any cluster center $v$ with at least one $u \in \mathcal{U} \cap \mathrm{Cl}(v)$ must receive any message from one such vertex.

Lemma 3.1. Up-cast and Down-cast can be implemented with $O\left(\frac{\log ^{3} n}{\beta \log (1 / \beta)}\right)$ calls to Local-Broadcast on $G$, in which each vertex participates in $O(\log n)$ Local-Broadcasts. I.e., the total time and energy per vertex are $O\left(\frac{\log ^{5} n}{\beta \log (1 / \beta)}\right)$ and $O\left(\log ^{3} n\right)$, respectively.

Proof. Consider the following two quantities:

$\mathcal{C}=O\left(\log _{(1 / \beta)} n\right)$. By Lemma 2.1, $\mathcal{C}$ is an upper bound on the number of clusters intersecting $N(v) \cup\{v\}$, with high probability. Intuitively, $\mathcal{C}$ represents the contention at $v$.

$\mathcal{D}=4 \log (n) / \beta$ is the maximum radius of any cluster, i.e., the maximum $\mathcal{L}$-value is at most $\mathcal{D}$.

If there were only one cluster, then doing an Up-cast or Down-cast would be easily reducible to $O(\log (n) / \beta)$ Local-Broadcasts. In order to minimize interference between neighboring clusters, we modify, slightly, the clustering algorithm so that all constituents of a cluster have shared randomness. When a new cluster center $v$ is formed, it generates a subset $S_{\mathrm{CI}(v)} \subset[\ell], \ell=\Theta(\mathcal{C} \log n)$, by including 
each index independently with probability $1 / \mathcal{C}$. It disseminates $S_{\mathrm{Cl}(v)}$ to all members of $\mathrm{Cl}(v)$ along with $\operatorname{ID}(\mathrm{Cl}(v))$. It is straightforward to show that with probability $1-1 / \operatorname{poly}(n)$, for every $v$,

$$
\text { There exists } j \in[\ell]: j \in S_{\mathrm{Cl}(v)} \text { and for all } u \in N(v), j \notin S_{\mathrm{Cl}(u)}
$$

Down-cast is implemented in $\mathcal{D}$ stages, each stage consisting of $\ell$ steps. In step $j$ of stage $i$, we execute Local-Broadcast with $\mathcal{S}$ consisting of every $v$ with a message to send such that $\mathcal{L}(v)=i-1$ and $j \in S_{\mathrm{CI}(v)}$, and with $\mathcal{R}$ consisting of every $u$ with $\mathcal{L}(u)=i$ and $j \in S_{\mathrm{Cl}(u)}$. By (2), during stage $i$, every layer- $i$ vertex in every participating cluster receives the cluster center's message with high probability. An Up-cast is performed in an analagous fashion.

Each Up-cast/Down-cast performs $\ell \mathcal{D}=\Theta(\mathcal{C D} \log n)=O\left(\frac{\log ^{3} n}{\beta \log (1 / \beta)}\right)$ Local-Broadcast on $G$, for a total of $O\left(\frac{\log ^{5} n}{\beta \log (1 / \beta)}\right)$ time. Each vertex $v$ participates in $O\left(\left|S_{\mathrm{Cl}(v)}\right|\right)$ Local-Broadcasts, which is $O(\log n)$ w.h.p., for a total of $O\left(\log ^{3} n\right)$ energy.

Lemma 3.2. A call to Local-Broadcast on the cluster graph $G^{*}=\operatorname{cluster}(G, \beta)$ can be simulated with $O\left(\frac{\log ^{3} n}{\beta \log (1 / \beta)}\right)$ calls to Local-Broadcast on $G$; each vertex in $V(G)$ participates in $O(\log n)$ Local-Broadcasts.

Proof. Let $\mathcal{S}$ and $\mathcal{R}$ be the sets of sending and receiving clusters in $G^{*}$. All members of $C$ know that $C$ is in $\mathcal{S}$ or $\mathcal{R}$. The Local-Broadcast algorithm has three steps.

1. Begin by doing a Down-cast in each $C \in \mathcal{S}$. Each member of $C$ learns the message $m_{C}$.

2. Perform one Local-Broadcast on $G$, with sender set $\bigcup_{C \in \mathcal{S}} C$ and receiver set $\bigcup_{C^{\prime} \in \mathcal{R}} C^{\prime}$. At this point, w.h.p., every $\mathcal{R}$-cluster adjacent to an $\mathcal{S}$-cluster has at least one constituent that has received a message.

3. Finally, do one Up-cast on every cluster $C \in \mathcal{R}$ to let the cluster center of $C$ learn one message from a constituent of $C$, if any.

The algorithm clearly satisfies the requirement of Local-Broadcast on cluster $(G, \beta)$. The number of calls to Local-Broadcast on $G$ is $O(\mathcal{C D} \log n)=O\left(\frac{\log ^{3} n}{\beta \log (1 / \beta)}\right)$ and each vertex participates in $O(\log n)$ of them.

\section{BFS with Sub-polynomial Energy}

\subsection{Technical Overview}

Suppose every vertex in the graph could cheaply compute its distance from the source up to an $\underline{\text { additive }} \pm \rho$ error. Given this knowledge, we could trivially solve exact BFS in $\tilde{O}(D)$ time and $\tilde{O}(\rho)$ energy per vertex, simply by letting vertices sleep through steps that they need not participate in. In particular, we would advance the BFS wavefront one layer at a time using calls to Local-Broadcast, except that each vertex $u$ would sleep through the first $\widetilde{\operatorname{dist}_{G}}(s, u)-\rho$ calls to Local-Broadcast, where $\widetilde{\operatorname{dist}_{G}}$ is the approximate distance. It would be guaranteed to fix $\operatorname{dist}_{G}(s, u)$ (and halt) in the next $2 \rho$ calls to Local-Broadcast.

Lemmas 2.2 and 2.3 suggest a method of obtaining approximate distances. If we computed the cluster graph $G^{*}=\operatorname{cluster}(G, \beta)$ and then computed exact distances on $G^{*}$, Lemmas 2.2 and 2.3 allow us to approximate all distances from the source, up to an additive error of $\tilde{O}\left(\beta^{-1}\right.$ ) (for small distances) and multiplicative error of $w^{2}$ (for larger distances), where $w=\Theta(\log n)$ is a sufficiently 
large multiple of $\log n$. Note that, from the perspective of energy efficiency, the main advantage to computing distances in $G^{*}$ rather than $G$ is that $G^{*}$ has a smaller diameter $w \beta \cdot \operatorname{diam}(G)$.

Our algorithm computes distances up to $D$ by advancing the BFS wavefront in $\lceil\beta D\rceil$ stages, extending the radius $\beta^{-1}$ per stage. The $i$ th wavefront $W_{i}$ is defined to be the vertex set

$$
W_{i}=\left\{u \in V(G) \mid \operatorname{dist}_{G}(S, u)=i \beta^{-1}\right\},
$$

where $S$ is the set of sources. (Recall that $\beta^{-1}$ is an integer.) To implement the $i$ th stage correctly it suffices to activate a vertex set $X_{i}$ that includes all the affected vertices, in particular:

$$
X_{i} \supset\left\{u \in V(G) \mid \operatorname{dist}_{G}(S, u) \in\left[i \beta^{-1},(i+1) \beta^{-1}\right]\right\} \quad \text { (w.h.p.) }
$$

In order for each vertex $u$ to decide whether it should join $X_{i}$ or sleep through the $i$ th stage, $u$ maintains lower and upper bounds on its distance to the $i$ th wavefront, or more accurately, the distance from its cluster $\mathrm{Cl}(u)$ to $W_{i}$ in $G$.

Invariant 4.1. Before the ith stage begins, each vertex $u$ knows $L_{i}(\mathrm{Cl}(u))$ and $U_{i}(\mathrm{Cl}(u))$ such that

$$
\operatorname{dist}_{G}\left(W_{i}, \mathrm{Cl}(u)\right)=\operatorname{dist}_{G}(S, \mathrm{Cl}(u))-i \beta^{-1} \in\left[L_{i}\left(\mathrm{Cl}(u), U_{i}(\mathrm{Cl}(u))\right] .\right.
$$

Clearly, if some cluster $C$ satisfies Invariant 4.1 at stage $i-1$ with the interval $\left[L_{i-1}(C), U_{i-1}(C)\right]$, it also satisfies Invariant 4.1 at stage $i$ with $L_{i}(C)=L_{i-1}(C)-\beta^{-1}$ and $U_{i}(C)=U_{i-1}(C)-\beta^{-1}$ since the $(i-1)$ th stage advances the wavefront by exactly $\beta^{-1}$. In the algorithm these are called Automatic Updates; they can be done locally, without expending any energy. In order to keep the interval $\left[L_{i}(C), U_{i}(C)\right]$ relatively narrow (and hence useful for keeping vertices in $C$ asleep), we occasionally refresh it with a Special Update. Let $W_{i}^{*} \subseteq V\left(G^{*}\right)$ be the clusters in $G^{*}$ that intersect the wavefront $W_{i}$. We call BFS on a subgraph $G_{i}^{*}$ of $G^{*}$ from the source-set $W_{i}^{*}$, up to a radius of $Z[i]$. The only clusters that participate in this recursive call are those that are likely to be relevant, i.e., those $C$ for which $L_{i}(C) \leq Z[i] \cdot \beta^{-1}$. (The $Z[i]$ sequence will be defined shortly.) After this recursive call completes we update $\left[L_{i}(C), U_{i}(C)\right]$ for all participating $C$ by applying Lemmas 2.2 and 2.3 to the (exact) distance $\operatorname{dist}_{G_{i}^{*}}\left(W_{i}^{*}, C\right)$ obtained in the cluster graph.

Specification. Our Recursive-BFS procedure (see Figure 2) takes four parameters: $G$, the graph, $S \subset V(G)$, the set of sources, $A \subseteq V(G)$, the set of active vertices (which is a superset of $S$ ), and $D$, the depth of the search. When we make a call to Recursive-BFS, every vertex can locally calculate $D$ and whether it is in $S$ or $A \sqrt[7]{ } G^{*}$ denotes the cluster graph returned by cluster $(G, \beta)$, where $\beta$ is a parameter fixed throughout the computation. We compute $G^{*}$ once, just before the first recursive call to Recursive-BFS $(G, \cdot, \cdot, \cdot)$; subsequent calls to Recursive-BFS on $G$ with different $(S, A, D)$ parameters can use the same $G^{*}$. It is important to remember that $G$ can be either the actual radio network $(\mathrm{RN})$ or a virtual $\mathrm{RN}$ on which we can simulate $\mathrm{RN}$ algorithms, with a certain overhead in terms of time and energy. At the termination of Recursive-BFS $(G, S, A, D)$, every vertex $u \in A$ returns $\operatorname{dist}_{A}(S, u)$ if it is at most $D$, and $\infty$ otherwise. Vertices in $V(G) \backslash A$ expend no energy.

Correctness. If one believes that the algorithm (Figure 2) faithfully implements the high level description given so far, its correctness is immediate. Every time we set $\left[L_{i}(C), U_{i}(C)\right]$ the interval is correct with probability $1-1 / \operatorname{poly}(n)$, either because $\left[L_{i-1}(C), U_{i-1}(C)\right]$ is correct (an Automatic

\footnotetext{
${ }^{7}$ The purpose of the $A$ parameter is to refrain from computing useless information. E.g., when we compute the distance from the clusters $W_{i}^{*}$ intersecting the $i$ th wavefront, we are only interested in distances to clusters intersecting as-yet unvisited vertices (those intersecting $A$ ), not settled vertices "behind" the wavefront.
} 
Recursive-BFS $(G, S, A, D)$

\section{[Initialize Distance Estimates]}

1. Call Recursive-BFS $\left(G^{*}, S^{*}, A^{*}, D^{*}\right)$ where $D^{*}=w \beta D$. For each cluster $C$ in $A^{*}$,

$$
L_{0}(C) \leftarrow \operatorname{dist}_{A^{*}}\left(S^{*}, C\right) \cdot \frac{1}{\beta w}, \quad U_{0}(C) \leftarrow \max \left\{w \beta^{-1}, w^{2} \cdot L_{0}(C)\right\} .
$$

2. $A=A \backslash\left\{u \mid L_{0}(\mathrm{Cl}(u))=\infty\right\} . \quad$ (Deactivate vertices at distance greater than $D$, w.h.p.)

3. For $i$ from 0 to $\lceil\beta D\rceil-1$

\section{[Iteratively Advance BFS Wavefront $\beta^{-1}$ Steps]}

4. Define $X_{i}=\left\{u \in A \mid L_{i}(\mathrm{Cl}(u)) \leq \beta^{-1}\right\}$.

5. Advance BFS wavefront from $W_{i}$ to $W_{i+1}$ using $\beta^{-1}$ calls to Local-Broadcast. Only vertices in $X_{i}$ participate in this step.

6. $A \leftarrow A \backslash\left\{u \mid \operatorname{dist}_{G}(S, u)<(i+1) \beta^{-1}\right\}$.

(Deactivate settled vertices.)

[Estimate Distances to $(i+1)$ th Wavefront $W_{i+1}$ ]

7. Define $G_{i+1}^{*}$ to be the subgraph of $G^{*}$ induced by

$$
\Upsilon=\left\{C \in A^{*} \mid L_{i}(C) \leq(Z[i+1]+1) \cdot \beta^{-1}\right\} .
$$

Vertices in $\Upsilon$-clusters participate in a Special Update.

Call Recursive-BFS $\left(G^{*}, W_{i+1}^{*}, \Upsilon, Z[i+1]\right)$.

For each cluster $C$ with $\operatorname{dist}_{G_{i+1}^{*}}\left(W_{i+1}^{*}, C\right)=x$, set

$$
\begin{aligned}
& L_{i+1}(C) \leftarrow \min \left\{Z[i+1] \cdot \beta^{-1}+1, x \cdot \beta^{-1} / w\right\}, \\
& U_{i+1}(C) \leftarrow \min \left\{U_{i}(C)-\beta^{-1}, \max \{x, 1\} \cdot \beta^{-1} w\right\} .
\end{aligned}
$$

8. Active vertices that did not participate in the Special Update perform an Automatic Update. For each $C \in A^{*} \backslash \Upsilon$,

$$
\begin{aligned}
& L_{i+1}(C) \leftarrow L_{i}(C)-\beta^{-1} \\
& U_{i+1}(C) \leftarrow U_{i}(C)-\beta^{-1}
\end{aligned}
$$

Figure 2: Recursive-BFS. 
Update), or because they are set according to Lemmas 2.2 and 2.3. which hold with probability $1-1 / \operatorname{poly}(n)$ (Special Update). If $L_{i}(C)$ is correct for all $C$, then $X_{i}$ will include all vertices necessary to compute the $(i+1)$ th wavefront, and the $i$ th stage will succeed, up to the $1 / \operatorname{poly}(n)$ error probability inherent in calls to Local-Broadcast. The main question is whether the procedure is efficient.

Efficiency. We will argue that for a very specific $Z[\cdot]$ sequence, which guides the Special Update steps, the following claims hold:

Claim 1. Each vertex is included in the set $X_{i}$ for $\tilde{O}(1)$ values of $i$.

Claim 2. For each vertex $u, \mathrm{Cl}(u)$ is included in $G_{i}^{*}$ for $\tilde{O}(1)$ values of $i$.

Our algorithms (cluster and Recursive-BFS) are based solely on calls to Local-Broadcast. Define $\operatorname{En}(D)$ to be the number of calls to Local-Broadcast that one vertex participates in when computing BFS to distance $D$. If Claims 1 and 2 hold, then

$$
\operatorname{En}(D) \leq \tilde{O}(1) \cdot \operatorname{En}(\tilde{O}(\beta D))+\tilde{O}\left(\beta^{-1}\right)
$$

The $\tilde{O}\left(\beta^{-1}\right)$ term accounts for the cost of computing $G^{*}$ (Lemma 2.5) and the $\tilde{O}(1)$ times a vertex is included in $X_{i}$ (Claim 11), each of which involves $\beta^{-1}$ Local-Broadcasts on $G$. Every recursive call to Recursive-BFS $\left(G^{*}, \cdot, \cdot, D^{\prime}\right)$ has $D^{\prime}=\tilde{O}(\beta D)$ and by Claim 2 each vertex participates in $\tilde{O}(1)$ such recursive calls. Moreover, according to Lemma 3.2, the energy overhead for simulating one call to Local-Broadcast on $G^{*}$ is $\tilde{O}(1)$ calls to Local-Broadcast on $G$. This justifies the first term of (3). The time and energy of our algorithm is analyzed in Theorem 4.1. As a foreshadowing of the analysis, if $D_{0}$ is the distance threshold of the top-level call to Recursive-BFS, we will set set $\beta=2^{-\sqrt{\log D_{0} \log \log n}}$ and apply (3) to recursion depth $\sqrt{\log D_{0} / \log \log n}$.

The Z-Sequence. The least obvious part of the Recursive-BFS algorithm is the $Z$-sequence, which guides how Special Updates are performed. Recall that $w=\Theta(\log n)$ is a sufficiently large multiple of $\log n$; if we are computing BFS to distance $D$ in $G$, then we need never compute BFS beyond distance $D^{*} \geq w \beta D$ in $G^{*}$. The $Z$-sequence is defined as follows.

$$
\begin{aligned}
Y[i] & =\max _{j \geq 0}\left\{2^{j} \text { such that } 2^{j} \mid i\right\} \\
\text { I.e., } Y & =(1,2,1,4,1,2,1,8,1,2,1,4,1,2,1,16,1,2,1,4,1,2,1,8,1,2,1,4,1,2,1,32, \ldots) \\
Z[0] & =D^{*} \\
Z[i] & =\min \left\{D^{*}, \alpha \cdot Y[i]\right\}, \quad \text { where } \alpha=4 \\
D^{*} & =\min _{j \geq 0}\left\{\alpha 2^{j} \text { such that } \alpha 2^{j} \geq w \beta D\right\}
\end{aligned}
$$

In other words, $Z$ is derived by multiplying $Y$ by $\alpha=4$, truncating large elements at $D^{*}$, and beginning the sequence at 0 , with $Z[0]=D^{*}$. (Here $Z[0]$ corresponds to the distance threshold $D^{*}$ used in Step 1 of Recursive-BFS to estimate distances to the 0th wavefront $W_{0}=S$.)

Figure 3 gives an example, from the perspective of a single cluster, of how the distance estimate evolve over time.

Organization of Section 4. In Section 4.2 we prove a number of lemmas that relate to the correctness and efficiency of Recursive-BFS, including proofs of Claims 11 and 2, In Section 4.3 we analyze the overall time and energy-efficiency of the BFS algorithm. 


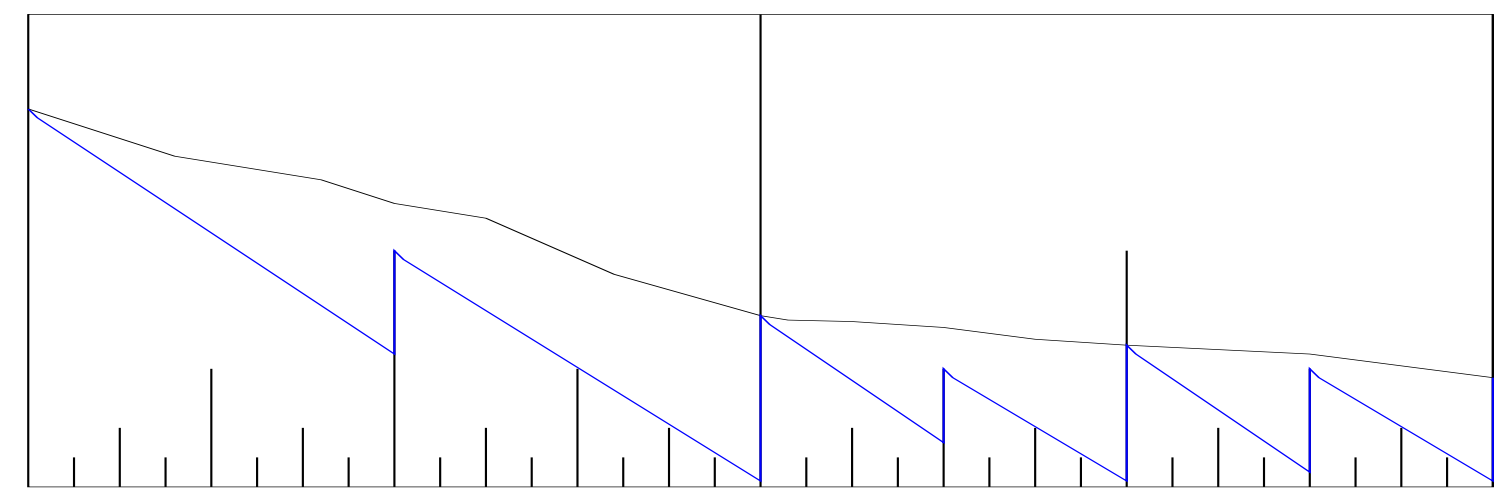

Figure 3: Part of the time evolution of the distance of a fixed cluster, $C$, from the frontier in the cluster graph $G^{*}$. The $x$-axis is time spent moving the wavefront across the underlying graph $G$. Every vertical tick mark is a time at which this is suspended, so that, recursively, BFS can be done on the cluster graph $G^{*}$, starting from the current wavefront. The height of each such tick mark indicates the depth to which this search is to be done. The $y$-axis is the distance of $C$ to the wave front, $\underline{i n} G^{*}$. The top curve shows the irregular, but monotonic, decrease of this distance over time. The bottom curve, in blue, shows the high-probability lower bound on this distance, from the perspective of the cluster in question. Note that every time the top curve intersects a tick mark, the cluster must participate in the BFS on the cluster graph, or this BFS will fail. Every time the bottom curve intersects a tick mark, the cluster will wake up in order to participate in the BFS, because it thinks it may be needed. Note that, by design, the lower curve often passes just above the tick marks without actually intersecting them. The reader should bear in mind that these two curves chart the actual/likely distance of $C$ to the wavefront in $G$; the algorithm maintains the related interval $\left[L_{i}(C), U_{i}(C)\right]$, which bounds distances from $C$ to the wavefront $\underline{i n} G$.

\subsection{Auxiliary Lemmas}

Lemma 4.1 justifies how distance estimates are updated in Steps 1, 7, and 8 of Recursive-BFS in order to preserve Invariant 4.1, with high probability.

Lemma 4.1. Let $W_{i}$ be the ith wavefront; let $\Upsilon$ include all clusters $C$ such that $\operatorname{dist}_{G}\left(W_{i}, C\right) \in$ $\left[i \beta^{-1},\left(i+Z^{\prime}\right) \beta^{-1}\right]$; and let $G_{i}^{*}$ be the subgraph of $G^{*}$ induced by $\Upsilon$. If $\mathrm{Cl}(u) \in \Upsilon$ and $\operatorname{dist}_{G}(S, u) \geq$ $i \beta^{-1}$, then w.h.p.,

$$
\operatorname{dist}_{G}\left(W_{i}, u\right) \in\left[\min \left\{\frac{Z^{\prime}}{\beta}+1, \operatorname{dist}_{G_{i}^{*}}\left(W_{i}^{*}, \mathrm{Cl}(u)\right) \cdot \frac{1}{w \beta}\right\}, \max \left\{1, \operatorname{dist}_{G_{i}^{*}}\left(W_{i}^{*}, \mathrm{Cl}(u)\right)\right\} \cdot \frac{w}{\beta}\right] .
$$

Proof. If $d=\operatorname{dist}_{G}\left(W_{i}, u\right) \geq Z^{\prime} \beta^{-1}+1$ then the lower bound is already correct, so suppose that $d \leq Z^{\prime} \beta^{-1}$. Let $P$ be any length- $d$ path from $u$ to $W_{i}$ in $G$. Lemma 2.2 implies that w.h.p., there is a path $P^{*}$ in $G_{i}^{*}$ from $W_{i}^{*}$ to $\mathrm{Cl}(u)$ with length at most $O(\beta d \log n)<w \beta d$, and so $\operatorname{dist}_{G_{i}^{*}}\left(W_{i}^{*}, \mathrm{Cl}(u)\right) \leq$ $w \beta d$, as required.

This upper bound follows from the cluster diameter upper bound $K=8 \log (n) / \beta \leq w /(2 \beta)-1$. Thus, if $\operatorname{dist}_{G_{i}^{*}}\left(W_{i}^{*}, \mathrm{Cl}(u)\right)=d^{\prime}$ then $\operatorname{dist}_{G}\left(W_{i}, u\right) \leq\left(d^{\prime}+1\right) \cdot(K+1) \leq \max \left\{d^{\prime}+1\right\} \cdot w \beta^{-1}$.

Lemma 4.1 shows that Step 1 of Recursive-BFS initializes $L_{0}(\cdot), U_{0}(\cdot)$ to satisfy Invariant 4.1 , w.h.p. Here $\Upsilon=A^{*}$ is the set of all active clusters; if $\operatorname{dist}_{A}(S, u) \in[0, D]$ (the relevant range), then Lemma 4.1 guarantees that $\operatorname{dist}_{A}(S, u) \in\left[L_{0}(\mathrm{Cl}(u)), U_{0}(\mathrm{Cl}(u))\right]$ after Step 1. The estimates set in Step 8 of Recursive-BFS are trivially correct; Lemma 4.1 also guarantees that the lower and upper bounds fixed in Step 7 are correct.

We use several properties of the $Z$ sequence, listed in Lemma 4.2. 
Lemma 4.2. Fix an index $i$.

1. For any number $b \geq \alpha$, define $j>i$ to be the smallest index such that $Z[j] \geq b$. Then

$$
j-i \leq b / \alpha \text {. }
$$

Suppose the number $b$ additionally satisfies that $b \leq Z[i]$ and $b \in\left\{\alpha, 2 \alpha, 4 \alpha, 8 \alpha, \ldots D^{*}\right\}$. Then we have $Z[i]=b$ and $j-i=Z[j] / \alpha$.

2. Define $j>i$ to be the smallest index such that $Z[j]>Z[i]$ or $Z[j]=D^{*}$. Then we have $j-i=Z[i] / \alpha$; moreover, all indices $k \in\{i+1, \ldots, j-1\}$ satisfy that $Z[k] \leq Z[i] / 2$.

Proof. Parts 1 and 2 follow from the fact that in the $Y$-sequence, the values at least $2^{\ell}$ appear periodically with period $2^{\ell}$. Thus, the values at least $\alpha 2^{\ell}$ in the $Z$-sequence also appear periodically with period $2^{\ell}$.

We are now prepared to prove Claim 1 .

Proof of Claim 1. It follows from Invariant 4.1 that $X_{i}$, as defined in Step 4 of Recursive-BFS, includes all active vertices within distance $\beta^{-1}$ of the $i$ th wavefront $W_{i}$. It remains to show no $u$ is included in $X_{i}$ for more than poly $(\log n)$ indices $i$.

Suppose that $u \in X_{i}$ for $i>0$. It follows that $L_{i}(\mathrm{Cl}(u)) \leq \beta^{-1}$ and that in the previous stage, $L_{i-1}(\mathrm{Cl}(u)) \leq 2 \beta^{-1}$. Since $Z[i] \geq \alpha=4$, it must have been that $\mathrm{Cl}(u)$ was included in $\Upsilon$ and participated in the Special Update (Step 7 of Recursive-BFS) before stage $i$. If $\operatorname{dist}_{G_{i}^{*}}\left(W_{i}^{*}, \mathrm{Cl}(u)\right)=x$ and after the Special Update, $L_{i}(\mathrm{Cl}(u)) \leq \beta^{-1}$, it must be that $x \leq w$, and hence $U_{i}(\mathrm{Cl}(u)) \leq w^{2} \beta^{-1}$. Thus, $u$ may participate in at most $w^{2}$ more stages (joining $X_{i}, X_{i+1}, \ldots, X_{i+w^{2}}$ ) before its distance is settled and it is deactivated, in Step 6 of Recursive-BFS.

Before proving Claim 2 we begin with three auxiliary lemmas, Lemmas 4.3, 4.4, and 4.5.

Lemma 4.3. Recall $\alpha=4$. Suppose cluster $C$ is included in $G_{i}^{*}$ and $G_{j}^{*}$, but not in $G_{i^{\prime}}^{*}$ for any $i^{\prime} \in\{i+1, \ldots, j-1\}$. Then we have

$$
\frac{L_{i}(C)}{8 \alpha} \leq \frac{j-i}{\beta} \leq \max \left\{\frac{1}{\beta}, \frac{L_{i}(C)}{\alpha}\right\}
$$

Proof. We prove the upper and lower bounds on $(j-i) / \beta$ separately.

Upper Bound. Select $j^{*}>i$ to be the first stage index for which $Z\left[j^{*}\right] \geq \min \left\{D^{*}, \beta L_{i}(C)\right\}$. Clearly $j^{*} \geq j$ since if $L_{i+1}(C), \ldots, L_{j^{*}}(C)$ were set according to Automatic Updates we would have $L_{j^{*}-1}(C) \leq L_{i}(C) \leq Z\left[j^{*}\right] \beta^{-1}$, which would trigger a Special Update to $L_{j^{*}}(C)$. There are two cases to consider, either of which establishes the upper bound on $(j-i) / \beta$.

- Suppose $\beta \cdot L_{i}(C)<\alpha$. Then $j=j^{*}=i+1$, and so $(j-i) / \beta=1 / \beta$.

- Suppose $\beta \cdot L_{i}(C) \geq \alpha$. According to Lemma 4.2(1), $j^{*}-i \leq \min \left\{D^{*}, \beta L_{i}(C)\right\} / \alpha$, and so $(j-i) / \beta \leq\left(j^{*}-i\right) / \beta \leq \min \left\{D^{*}, \beta L_{i}(C)\right\} /(\alpha \beta) \leq L_{i}(C) / \alpha$. 
Lower Bound. In order to prove that $(j-i) \cdot \beta^{-1} \geq L_{i}(C) /(8 \alpha)$ it suffices to find any particular index $j^{*}$ such that:

1. $\left(j^{*}-i\right) \cdot \beta^{-1} \geq L_{i}(C) /(8 \alpha)$.

2. For all $j^{\prime} \in\left[i+1, j^{*}\right], C$ is not included in $G_{j^{\prime}}^{*}$.

Condition 2 implies that $j^{\star}<j$ and then Condition 1 implies that $(j-i) / \beta>\left(j^{*}-i\right) \cdot \beta^{-1} \geq$ $L_{i}(C) /(8 \alpha)$, as desired. We will explain how to select $j^{*}$ shortly. In the meantime, consider the following two conditions; we will argue that (a) and (b) imply Condition 2 above.

(a) For all $j^{\prime} \in\left[i+1, j^{*}-1\right]$, we have $Z\left[j^{\prime}\right]<Z\left[j^{*}\right]$.

(b) $L_{i}(C)-\left(j^{*}-i\right) \cdot \beta^{-1}>Z\left[j^{*}\right] \cdot \beta^{-1}$.

Recall that $C$ is not included in $G_{j^{\prime}}^{*}$ iff $L_{j^{\prime}-1}(C)>\left(Z\left[j^{\prime}\right]+1\right) \cdot \beta^{-1}$, so it suffices to prove the latter inequality for every $j^{\prime} \in\left[i+1, j^{*}\right]$. By induction, we can assume that the claim is true for all $j^{\prime \prime} \in\left[i+1, j^{\prime}-1\right]$, i.e., $L_{j^{\prime \prime}}(C)$ was set according to an Automatic Update (Step 8) and $L_{j^{\prime \prime}}(C)=L_{i}(C)-\left(j^{\prime \prime}-i\right) \cdot \beta^{-1}$. Thus,

$$
\begin{aligned}
L_{j^{\prime}-1}(C) & =L_{i}(C)-\left(\left(j^{\prime}-1\right)-i\right) \cdot \beta^{-1} & & \text { Follows from induction hypothesis } \\
& \geq L_{i}(C)-\left(\left(j^{*}-1\right)-i\right) \cdot \beta^{-1} & & \\
& >\left(Z\left[j^{*}\right]+1\right) \cdot \beta^{-1} & & \text { by (b) } \\
& >\left(Z\left[j^{\prime}\right]+1\right) \cdot \beta^{-1} & & \text { by (a) }
\end{aligned}
$$

Choice of $j^{*}$. Select $x$ to be the integer in $\left\{\alpha, 2 \alpha, 4 \alpha, 8 \alpha, \ldots, D^{*}\right\}$ such that

$$
x \in\left[\frac{\beta \cdot L_{i}(C)}{8}, \frac{\beta \cdot L_{i}(C)}{4}\right) .
$$

It is guaranteed that $x$ exists so long as $\beta \cdot L_{i}(C)>4 \alpha$. When $\beta \cdot L_{i}(C) \leq 4 \alpha$, we already have the desired lower bound on $(j-1) \cdot \beta^{-1}$ since $L_{i}(C) /(8 \alpha) \leq \beta^{-1} / 2<\beta^{-1} \leq(j-i) \cdot \beta^{-1}$.

Observe that $Z[i]$, like $x$, is also an integer in $\left\{\alpha, 2 \alpha, 4 \alpha, 8 \alpha, \ldots, D^{*}\right\}$. In a Special Update, the largest value that $L_{i}(C)$ can attain is $Z[i] \cdot \beta^{-1}+1$, hence

$$
Z[i] \geq \beta \cdot\left(L_{i}(C)-1\right)>\beta \cdot L_{i}(C) / 2>2 x,
$$

Define $j^{*}>i$ to be the smallest index such that $Z\left[j^{*}\right] \geq x$. In particular, since $Z[i] \geq 2 x>x$, Lemma 4.2(1) guarantees that $Z\left[j^{*}\right]=x$ and hence

$$
j^{*}-i=Z\left[j^{*}\right] / \alpha=x / \alpha \geq \beta \cdot L_{i}(C) /(8 \alpha) .
$$

Thus Condition 1 is met for this choice of $j^{*}$.

Condition (a) is also met, since by definition of $j^{*}, Z\left[j^{\prime}\right]<x=Z\left[j^{*}\right]$ for all $j^{\prime} \in\left[i+1, j^{*}-1\right]$. Now we turn to Condition (b). Observe that

$$
j^{*}-i=Z\left[j^{*}\right] / \alpha=x / \alpha<\beta \cdot L_{i}(C) /(4 \alpha) .
$$


We prove that $L_{i}(C)-\left(j^{*}-i\right) \cdot \beta^{-1}>Z\left[j^{*}\right] \cdot \beta^{-1}$.

$$
\begin{aligned}
Z\left[j^{*}\right] \cdot \beta^{-1} & <2 x \cdot \beta^{-1} \\
& <L_{i}(C) / 2 \\
& =L_{i}(C)(1-2 / \alpha) \\
& <L_{i}(C)-8\left(j^{*}-i\right) \cdot \beta^{-1} \\
& <L_{i}(C)-\left(j^{*}-i\right) \cdot \beta^{-1}
\end{aligned}
$$

since $Z\left[j^{*}\right]<2 x$

since $x \in\left[\beta \cdot L_{i}(C) / 8, \beta \cdot L_{i}(C) / 4\right)$

since $\alpha=4$

by (4), $\left(j^{*}-i\right) \cdot \beta^{-1}<L_{i}(C) /(4 \alpha)$

Conditions (a) and (b) imply Condition 2, which implies $L_{i}(C) /(8 \alpha) \leq(j-i) \cdot \beta^{-1}$.

Lemma 4.4. Suppose $C$ appears in $G_{i}^{*}$ and $G_{j}^{*}$ but not in $G_{i^{\prime}}^{*}$ for any $i^{\prime} \in\{i+1, \ldots, j-1\}$. Suppose that when $L_{i}(C)$ is set during a Special Update (Step 7 of Recursive-BFS), we have $L_{i}(C)=$ $(Z[i] / \beta)+1$. It must be that $Z[j]>Z[i]$ or $Z[j]=D^{*}$.

Proof. Define $j^{*}>i$ to be the smallest index such that $Z\left[j^{*}\right]>Z[i]$ or $Z\left[j^{*}\right]=D^{*}$. To prove the lemma it suffices to show that $j^{*}=j$, i.e., $L_{j^{\prime}}(C)$ is set according to an Automatic Update for $j^{\prime} \in\left\{i+1, \ldots, j^{*}-1\right\}$ but $C$ appears in $G_{j^{*}}$ and participates in a Special Update.

To prove that $L_{j^{\prime}}(C)$ is set according to an Automatic Update (assuming, inductively, that the claim holds for $\left.L_{i+1}(C), \ldots, L_{j^{\prime}-1}(C)\right)$ it suffices to show

$$
L_{j^{\prime}-1}(C)-\beta^{-1}=L_{i}(C)-\left(j^{\prime}-i\right) \cdot \beta^{-1}>Z[k] \cdot \beta^{-1} .
$$

By Lemma 4.2(2), $j^{*}-i=Z[i] / \alpha$. Since $j^{\prime}<j^{*}$ we have

$$
\left(j^{\prime}-i\right) \cdot \beta^{-1}<\left(j^{*}-i\right) \cdot \beta^{-1}=Z[i] \cdot \beta^{-1} / \alpha<L_{i}(C) / \alpha .
$$

It follows that

$$
L_{i}(C)-\left(j^{\prime}-i\right) \cdot \beta^{-1}>(1-1 / \alpha) L_{i}(C)>L_{i}(C) / 2 .
$$

On the other hand, Lemma 4.2(2) implies that

$$
Z\left[j^{\prime}\right] \cdot \beta^{-1} \leq(Z[i] / 2) \cdot \beta^{-1}<L_{i}(C) / 2 .
$$

Therefore $L_{i}(C)-\left(j^{\prime}-i\right) \beta^{-1}>Z\left[j^{\prime}\right] \cdot \beta^{-1}$, implying $L_{j^{\prime}}(C)$ is set according to an Automatic Update. Finally, from the definition of $i$ and $j^{*}$ we have

$$
L_{j^{*}-1}(C)<L_{i}(C)=Z[i] \cdot \beta^{-1}+1 \leq Z\left[j^{*}\right] \cdot \beta^{-1}+1<\left(Z\left[j^{*}\right]+1\right) \cdot \beta^{-1},
$$

meaning $C$ appears in $G_{j^{*}}^{*}$ and $L_{j^{*}}(C)$ is set according to a Special Update.

In the Recursive-BFS algorithm, the upper bound estimates $U_{i}(C)$ are all monotonically decreasing with $i$, due to the way Special and Automatic Updates are performed in Steps 7 and 8. On the other hand, the lower bound estimates $L_{i}(C)$ are only monotonically decreasing during Automatic Updates and may oscillate many times over the execution of the algorithm. (See Figure 3 for a depiction of how this happens.) Since $U$.(.)-values offer a more stable way to measure progress, we need to connect them with the L.(.)-values, which directly influence the composition of $X_{i}$ and $G_{i}^{*}$.

Lemma 4.5. If $\left[L_{i}(C), U_{i}(C)\right]$ is set during a Special Update step, then

$$
U_{i}(C) \leq \max \left\{2 w^{2} \cdot L_{i}(C), 2 w^{2} \cdot \beta^{-1}\right\}
$$


Proof. The proof is by induction on $i$. We regard Step 1 of Recursive-BFS as the Special Update for $i=0$. The claim clearly holds for $i=0$ since $U_{0}(C)$ is set such that $U_{0}(C) \in\left\{w \beta^{-1}, w^{2} \cdot L_{0}(C)\right\}$. Assume, inductively, that the lemma holds for all indices less than $i$.

In general, whenever $L_{i}(C)$ is set to be $x \beta^{-1} / w$ in Step 7 , where $x=\operatorname{dist}_{G_{i}^{*}}\left(W_{i}^{*}, C\right)$, the claim holds since $U_{i}(C) \in\left\{w \beta^{-1}, w^{2} \cdot L_{i}(C)\right\}$. Thus, we may proceed under the assumption that $L_{i}(C)$ is set to be $Z[i] \cdot \beta^{-1}+1$ during a Special Update.

Define $i^{*}<i$ to be the last stage in which $L_{i^{*}}(C)$ was set by a Special Update. We consider two cases, depending on how $L_{i^{*}}(C)$ was set.

- Suppose $L_{i^{*}}(C)$ is set to be $x /(\beta w) \leq Z\left[i^{*}\right] / \beta$ in the Special Update, and as a consequence, $U_{i^{*}}(C) \leq \max \left\{w \beta^{-1}, w^{2} \cdot L_{i^{*}}(C)\right\}$. (Here $x>0$ is the BFS-label of $C$ found in Step 7.) If $U_{i^{*}}(C) \leq\left(2 w^{2}+1\right) \cdot \beta^{-1}$, then we are already done, since $U_{i}(C) \leq U_{i^{*}}(C)-1 / \beta \leq 2 w^{2} \cdot \beta^{-1}$. Thus, we may assume $U_{i^{*}}(C)>\left(2 w^{2}+1\right) \cdot \beta^{-1}$, and consequently, that $L_{i^{*}}(C)>2 \beta^{-1}$.

By Lemma 4.3, we have $\left(i-i^{*}\right) / \beta \leq \max \left\{1 / \beta, L_{i^{*}}(C) / \alpha\right\}<L_{i^{*}}(C) / 2$. In order for $L_{i}(C)$ to be set by a Special Update, it is necessary that $L_{i-1}(C) \leq(Z[i]+1) \cdot \beta$. Thus, we must have

$$
\begin{aligned}
Z[i] \cdot \beta^{-1} & \geq L_{i-1}(C)-\beta^{-1} & & \text { since } L_{i-1}(C) \leq(Z[i]+1) \cdot \beta^{-1} \\
& =L_{i^{*}}(C)-\left(i-i^{*}\right) \cdot \beta^{-1} & & \text { since } C \text { does not appear in } G_{i^{*}+1}^{*}, \ldots, G_{i-1}^{*} \\
& \geq L_{i^{*}}(C) / 2 & & \text { since }\left(i-i^{*}\right) \cdot \beta^{-1} \leq L_{i^{*}}(C) / 2
\end{aligned}
$$

Remember that $L_{i}(C)=Z[i] \cdot \beta^{-1}+1$, and based on this we show that $U_{i}(C) \leq 2 w^{2} \cdot L_{i}(C)$.

$$
\begin{aligned}
L_{i}(C) & =Z[i] \cdot \beta^{-1}+1 & & \\
& >L_{i^{*}}(C) / 2 & & \text { since } Z[i] \cdot \beta^{-1} \geq L_{i^{*}}(C) / 2 \\
& \geq U_{i^{*}}(C) /\left(2 w^{2}\right) & & \text { since } U_{i^{*}}(C) \leq w^{2} L_{i^{*}}(C) \\
& >U_{i}(C) /\left(2 w^{2}\right) & & \text { since } U_{i}(C)<U_{i^{*}}(C), \text { as } i^{*}<i .
\end{aligned}
$$

- Now consider the case when $L_{i^{*}}(C)$ is set to be $Z\left[i^{*}\right] \cdot \beta^{-1}+1$. By Lemma 4.4, we have $Z[i] \geq Z\left[i^{*}\right]$. Therefore, $L_{i}(C)=Z[i] \cdot \beta^{-1}+1 \geq Z\left[i^{*}\right] \cdot \beta^{-1}+1=L_{i^{*}}(C)$. By the inductive hypothesis, it is guaranteed that $U_{i^{*}}(C) \leq \max \left\{2 w^{2} \cdot \beta^{-1}, 2 w^{2} \cdot L_{i^{*}}(C)\right\}$. If $U_{i^{*}}(C) \leq 2 w^{2} \cdot \beta^{-1}$, then we are done. If $U_{i^{*}}(C) \leq 2 w^{2} \cdot L_{i^{*}}(C)$, then we have

$$
L_{i}(C) \geq L_{i^{*}}(C) \geq U_{i^{*}}(C) /\left(2 w^{2}\right)>U_{i}(C) /\left(2 w^{2}\right) .
$$

This concludes the induction and the proof.

We are now in a position to prove Claim 2, that each vertex participates in $G_{i}^{*}$ for at most $\tilde{O}(1)$ indices $i$.

Proof of Claim 2. Suppose that $C$ participates in a Special Update that sets $\left[L_{i}(C), U_{i}(C)\right]$ with $U_{i}(C) \geq 2 w^{2} \cdot \beta^{-1}$ and that the next interval to be set by a Special Update is $\left[L_{j}(C), U_{j}(C)\right]$. Then

$$
(j-i) \geq \frac{\beta \cdot L_{i}(C)}{8 \alpha} \geq \frac{\beta \cdot U_{i}(C)}{16 \alpha w^{2}} .
$$

The first inequality of (5) follows from Lemma 4.3 and the second inequality from Lemma 4.5. Since $U_{*}(C)$ is decremented by at least $\beta^{-1}$ in each stage, (5) implies that

$$
U_{j}(C) \leq U_{i}(C)-(j-i) \cdot \beta^{-1} \leq U_{i}(C)\left(1-\frac{1}{16 \alpha w^{2}}\right) .
$$


In other words, $C$ participates in at $\operatorname{most} \log _{1+\Theta\left(1 / w^{2}\right)} D=\Theta\left(w^{2} \log D\right)=O\left(\log ^{3} n\right)$ Special Updates until some stage $i$ in which $U_{i}(C)<2 w^{2} \cdot \beta^{-1}$, after which $C$ participates in at most $O\left(w^{2}\right)$ Special Updates all constituents of $C$ settle their distance from the source and are deactivated.

\subsection{Time and Energy Complexity of BFS}

The remainder of this section constitutes a proof of Theorem 4.1 ,

Theorem 4.1. Let $G=(V, E)$ be a radio network, $s \in V$ be a distinguished source vertex, and $D=\max _{u} \operatorname{dist}_{G}(s, u)$. A Breadth First Search labeling can be computed in $\tilde{O}(D) \cdot 2^{O(\sqrt{\log D \log \log n})}$ time and $\tilde{O}(1) \cdot 2^{O(\sqrt{\log D \log \log n})}$ energy, with high probability.

The main problem is to compute BFS up to some threshold distance $D_{0}$. Once we have a solution to this problem, we can obtain bounds in terms of the (unknown) $D$ parameter by testing every $D_{0}=2^{k}$ that is a power of 2 , stopping at the first value that labels all of $V(G)$. We use a call to Local-Broadcast as a unit of measurement of both time and energy, i.e., calling Local-Broadcast takes one unit of time, and every participating vertex expends one unit of energy. (By Lemma 2.4 actual time and energy are at most a $O\left(\log ^{2} n\right)$ factor larger.)

The algorithm we apply is a slightly modified Recursive-BFS, where all cluster graphs in all recursive invocations are constructed with $\beta=2^{-\sqrt{\log D_{0} \log \log n}}$. We only apply Recursive-BFS to recursion depth $L=\sqrt{\log D_{0} / \log \log n}$, at which point we revert to the trivial BFS algorithm that settles all distances up to $D^{\prime}$ using $D^{\prime}$ time and energy, by calling Local-Broadcast $D^{\prime}$ times.

Define $\operatorname{En}_{r}\left(D^{\prime}\right)$ to be the number of calls to Local-Broadcast that a vertex participates in when computing BFS to distance $D^{\prime}$, and when the recursion depth is $r \in[0, L]$. Thus, we have

$$
\operatorname{En}_{r}\left(D^{\prime}\right)= \begin{cases}\tilde{O}(1) \cdot \operatorname{En}_{r+1}\left(\tilde{O}\left(\beta D^{\prime}\right)\right)+\tilde{O}\left(\beta^{-1}\right) & \text { if } r<L \\ D^{\prime} & \text { if } r=L\end{cases}
$$

By Lemma 2.5 the cost to create the cluster graph $G^{*}$ is $\tilde{O}\left(\beta^{-1}\right)$. By Claim 1 each vertex appears in $X_{i}$ for $\tilde{O}(1)$ stages $i$, and for each, participates in $\beta^{-1}$ calls to Local-Broadcast. These costs are covered by the $\tilde{O}\left(\beta^{-1}\right)$ term. All calls to Recursive-BFS on $G^{*}$ involve computing BFS to some distance at most $D^{*}=w \beta D^{\prime}=\tilde{O}\left(\beta D^{\prime}\right)$. By Claim 2, every vertex participates in $\tilde{O}(1)$ such recursive calls. Moreover, by Lemma 3.2, every cluster $C$ (vertex in $G^{*}$ ) that participates in a call to Local-Broadcast on $G^{*}$ can be simulated such that constituent vertices of $C$ participate in $\tilde{O}(1)$ calls to Local-Broadcast on $G$. The costs of recursive calls are represented by the $\tilde{O}(1) \cdot \operatorname{En}_{r+1}\left(\tilde{O}\left(\beta D^{\prime}\right)\right)$ term.

When the recursion depth $r$ reaches $L$, the maximum value of $D^{\prime}$ is therefore at most

$$
D_{L}=D_{0} \cdot(\tilde{O}(\beta))^{L}=(\tilde{O}(1))^{L}=2^{O\left(\sqrt{\log D_{0} \log \log n}\right)},
$$

since $\beta^{L}=D_{0}^{-1}$. Thus, the energy cost of the top-level recursive call is at most

$$
\operatorname{En}_{0}\left(D_{0}\right)=(\tilde{O}(1))^{L} \cdot\left(D_{L}+\tilde{O}\left(\beta^{-1}\right)\right)=\tilde{O}(1) \cdot 2^{O\left(\sqrt{\log D_{0} \log \log n}\right)}
$$

We can set up a similar recursive expression for the time of this algorithm.

$$
\operatorname{Time}_{r}\left(D^{\prime}\right) \leq \begin{cases}O\left(D^{\prime}\right)+\tilde{O}\left(\beta^{-1}\right) \cdot \sum_{i=0}^{\left\lceil\beta D^{\prime}\right\rceil-1} \mathrm{Time}_{r+1}(Z[i]) & \text { if } r<L \\ D^{\prime} & \text { if } r=L\end{cases}
$$


The $r=L$ case is the time of the trivial algorithm, so we focus on justifying the expression for $r<L$. The time to advance the BFS wavefront over all $\left\lceil\beta D^{\prime}\right\rceil$ stages of Step 5 is $O\left(D^{\prime}\right)$. We treat Step 1 as the Special Update for $i=0$ with $Z[0]=D^{*}$. In general, the Special Update for stage $i$ takes $\operatorname{Time}_{r+1}(Z[i])$ time with respect to $G^{*}$, and each unit of time (i.e., a call to Local-Broadcast) is simulated in $G$ in time linear in the maximum cluster diameter, namely $\tilde{O}\left(\beta^{-1}\right)$. By Lemma 4.2, each value $b \in B=\left\{\alpha, 2 \alpha, 4 \alpha, \ldots, D^{*}\right\}$ appears less than $\left(\beta D^{\prime} / b\right)$ times in $Z[0], \ldots, Z\left[\left\lceil\beta D^{\prime}\right\rceil-1\right]$, hence we can rewrite the sum as $\sum_{b \in B}\left(\beta D^{\prime} / b\right) \cdot \operatorname{Time}_{r+1}(b)$. Assuming inductively that $\operatorname{Time}_{r+1}(b)$ is $b \cdot(\tilde{O}(1))^{L-(r+1)}$, which holds when $r+1=L$, we have

$$
\begin{aligned}
\operatorname{Time}_{r}\left(D^{\prime}\right) & \leq O\left(D^{\prime}\right)+\tilde{O}\left(\beta^{-1}\right) \cdot \sum_{b \in B}\left(\beta D^{\prime} / b\right) \cdot \text { Time }_{r+1}(b) \\
& =O\left(D^{\prime}\right)+\tilde{O}(1) \cdot \sum_{b \in B}\left(D^{\prime} / b\right) \cdot b \cdot(\tilde{O}(1))^{L-(r+1)} \\
& =D^{\prime} \cdot(\tilde{O}(1))^{L-r}
\end{aligned}
$$

Hence $\operatorname{Time}_{0}\left(D_{0}\right)=D_{0} \cdot(\tilde{O}(1))^{L}=\tilde{O}\left(D_{0}\right) \cdot 2^{O\left(\sqrt{\log D_{0} \log \log n}\right)}$.

\section{Hardness of Diameter Approximation}

In this section, we show that certain approximations of diameter cannot be computed in $o(n)$ energy, even allowing messages of unlimited size. Our lower bounds also hold in the setting where the network supports collision detection, i.e., in each time slot $t$, each listener $v$ is able to distinguish between the following two cases: (i) at least two vertices in $N(v)$ transmit at time $t$ (noise), or (ii) no vertex in $N(v)$ transmits at time $t$ (silence).

First, we show that computing a $(2-\epsilon)$-approximation of diameter is hard by proving that it takes $\Omega(n)$ energy to distinguish between (i) an $n$-vertex complete graph $K_{n}$ (which has diameter 1 ), or (ii) an $n$-vertex complete graph minus one edge $K_{n}-e$ (which has diameter 2 ).

Theorem 5.1. The energy complexity of computing a $(2-\epsilon)$-approximation of diameter is $\Omega(n)$, even on the class of unit-disc graphs.

Proof. Throughout the proof, we consider the scenario where the underlying graph is $K_{n}$ with probability $1 / 2$, and is $K_{n}-e$ with probability $1 / 2$. The edge $e$ is chosen uniformly at random. Observe that both $K_{n}$ and $K_{n}-e$ are both unit disc graphs. Let $\mathcal{A}$ be any randomized algorithm that is able to distinguish between $K_{n}$ and $K_{n}-e$. We make the following simplifying assumptions, which only increase the capabilities of the vertices.

- Each vertex has a distinct ID from $[n]$.

- All vertices have access to a shared random string.

- By the end of each time slot $t$, each vertex knows the following information: (i) the IDs of the vertices transmitting at time $t$, (ii) the IDs of the vertices listening at time $t$, and (iii) the channel feedback (i.e., noise, silence, or a message $m$ ) for each listening vertex.

With the above extra capabilities, all vertices share the same history. Since the actions of the vertices at time $t+1$ depend only on the shared history of all vertices and their shared random bits, by the end of time $t$ all vertices are able to predict the actions (i.e., transmit a message $m$, listen, or idle) of all vertices at time $t+1$. 
We say that time $t$ is good for a pair $\{u, v\}$ if the following conditions are met. Intuitively, if $t$ is not good for $\{u, v\}$, then what happens at time $t$ does not reveal any information as to whether $\{u, v\}$ is an edge.

- The number of transmitting vertices at time $t$ is either 1 or 2 ,

- One of the two vertices $\{u, v\}$ listens at time $t$, and the other one transmits at time $t$.

Once the shared random string is fixed, define $X_{\text {bad }}$ to be the set of pairs $\{u, v\}$ such that there is no time $t$ that is good for $\{u, v\}$ in an execution of $\mathcal{A}$ on $K_{n}$. Define $X_{\text {good }}$ to be the remaining pairs.

We claim that if the energy per vertex is at most $E=(n-1) / 8$, then for every pair $\{u, v\}$, $\mathbf{P}\left(\{u, v\} \in X_{\text {bad }}\right) \geq 1 / 2$. Recall that if a time $t$ is good for some pair, then the number of transmitting vertices is at most 2. Thus, if $t$ is good for $x$ pairs, then at least $x / 2$ vertices listen at time $t$, and so the total energy spent over all vertices and all time slots is at least $\left|X_{\text {good }}\right| / 2$. On the other hand, it is also at most $n E=n(n-1) / 8$. If $n(n-1) / 8 \geq\left|X_{\text {good }}\right| / 2$, then $\left|X_{\text {bad }}\right| \geq n(n-1) / 4$ and $\mathbf{P}\left(\{u, v\} \in X_{\text {bad }}\right) \geq 1 / 2$.

Recall that we pick $e$ at random and then choose the input graph to be either $K_{n}$ or $K_{n}-e$. Once $e$ is selected, let $\mathcal{E}$ be the event that $e \in X_{\text {bad }}$, which now depends only on the shared random string. When $\mathcal{E}$ occurs, the execution of $\mathcal{A}$ is identical on both $K_{n}$ and $K_{n}-e$, and so the success probability of $\mathcal{A}$ is at most $1 / 2$. Thus, $\mathcal{A}$ fails with probability at least $(1 / 2) \mathbf{P}(\mathcal{E}) \geq 1 / 4$. This contradicts the assumption that $\mathcal{A}$ is able to distinguish between $K_{n}$ and $K_{n}-e$.

For sparse graphs (i.e., those with $O(\log n)$-arboricity), we show that $(3 / 2-\epsilon)$-approximation of diameter is hard. The proof follows the framework of [1], which shows that computing diameter takes $\Omega\left(n / \log ^{2} n\right)$ time in the CONGEST model, or more generally $\Omega\left(\frac{n}{B \log n}\right)$ time in the messagepassing model with $B$-bit message size constraint. Note that a time lower bound in CONGEST does not, in general, imply any lower bound in $\mathrm{RN}[\infty]$, which has no message size constraint. The main challenge for proving Theorem 5.2 is that we allow messages of unbounded length.

Theorem 5.2. The energy complexity of computing an $(3 / 2-\epsilon)$-approximation of diameter is $\Omega\left(n / \log ^{2} n\right)$, even on graphs of $O(\log n)$-arboricity or $O(\log n)$ treewidth.

Proof. The proof is based on a reduction from the set-disjointness problem of communication complexity, which is defined as follows. Consider two players $A$ and $B$, each of them holding a subset of $\{0, \ldots, n-1\}$. Their task is to decide whether their subsets are disjoint. If the maximum allowed failure probability is $f<1 / 2$, then they need to communicate $\Omega(n)$ bits [8, 26]. This is true even if the two players have access to a public random string.

Lower Bound Graph Construction. Let $S_{A}=\left\{a_{1}, \ldots, a_{\alpha}\right\}$ and $S_{B}=\left\{b_{1}, \ldots, b_{\beta}\right\}$ be two subsets of $\{0, \ldots, k-1\}$ corresponding to an instance of set-disjointness problem. We assume that $k=2^{\ell}$, for some positive integer $\ell$, and so each element $s \in S_{A} \cup S_{B}$ is represented as a binary string of length $\ell=\log k$. We write $\operatorname{Ones}(s) \subseteq[\ell]=\{1, \ldots, \ell\}$ to denote the set of indices $i$ in $[\ell]$ such that $s[i]=1$ (i.e., the $i$ th bit of $s$ is 1 ); similarly, $\operatorname{Zeros}(s)=[\ell] \backslash \operatorname{Ones}(s)$ is the set of indices $i$ in $[\ell]$ such that $s[i]=0$. For example, if the binary representation of $s$ is $10110010(\ell=8)$, then Ones $(s)=\{1,3,4,7\}$ and $\operatorname{Zeros}(s)=\{2,5,6,8\}$.

Define the graph $G=(V, E)$ as follows.

Vertex Set. Define $V=V_{A} \cup V_{B} \cup V_{C} \cup V_{D} \cup\left\{u^{\star}, v^{\star}\right\}$, where $V_{A}=\left\{u_{1}, \ldots, u_{\alpha}\right\}, V_{B}=\left\{v_{1}, \ldots, v_{\beta}\right\}$, $V_{C}=\left\{w_{1}, \ldots, w_{\ell}\right\}$, and $V_{D}=\left\{x_{1}, \ldots, x_{\ell}\right\}$. Note that we have natural 1-1 correspondences $V_{A} \leftrightarrow S_{A}, V_{B} \leftrightarrow S_{B}, V_{C} \leftrightarrow[\ell]$, and $V_{D} \leftrightarrow[\ell]$. 
Edge Set. The edge set $E$ is constructed as follows. Initially $E=\emptyset$.

For each vertex $u_{i} \in V_{A}$ and each $w_{j} \in V_{C}$, add $\left\{u_{i}, w_{j}\right\}$ to $E$ if $j \in \operatorname{Ones}\left(a_{i}\right)$.

For each vertex $u_{i} \in V_{A}$ and each $x_{j} \in V_{D}$, add $\left\{u_{i}, x_{j}\right\}$ to $E$ if $j \in \operatorname{Zeros}\left(a_{i}\right)$.

For each vertex $v_{i} \in V_{B}$ and each $w_{j} \in V_{C}$, add $\left\{v_{i}, w_{j}\right\}$ to $E$ if $j \in \operatorname{Zeros}\left(b_{i}\right)$.

For each vertex $v_{i} \in V_{B}$ and each $x_{j} \in V_{D}$, add $\left\{v_{i}, x_{j}\right\}$ to $E$ if $j \in \operatorname{Ones}\left(b_{i}\right)$.

Add edges between $u^{\star}$ and all vertices in $V_{A} \cup V_{C} \cup V_{D}$.

Add edges between $v^{\star}$ and all vertices in $V_{B} \cup V_{C} \cup V_{D}$.

The graph $G$ has $n=\alpha+\beta+2 \ell+2 \leq 2(k+\log k+1)$ vertices. It is straightforward to show that $G$ has arboricity and treewidth $O(\log k)=O(\log n)$.

A crucial observation is that if $S_{A} \cap S_{B}=\emptyset$ (a yes-instance for the set-disjointness problem), then the diameter of $G$ is 2 ; otherwise (a $n o$-instance for the set-disjointness problem) the diameter of $G$ is 3. This can be seen as follows. First of all, observe that we must have $\operatorname{dist}(s, t) \leq 2$ unless $s \in V_{A}$ and $t \in V_{B}$. Now suppose $s=u_{i} \in V_{A}$ and $t=v_{j} \in V_{B}$.

- Consider the case $a_{i} \neq b_{j}$. We show that $\operatorname{dist}(s, t)=2$. Note that there is an index $l \in[\ell]$ such that $a_{i}$ and $b_{j}$ differ at the $l$ th bit. If the $l$ th bit of $a_{i}$ is 0 and the $l$ th bit of $b_{j}$ is 1 , then $\left(u_{i}, x_{l}, v_{j}\right)$ is a length-2 path between $s$ and $t$. If the $l$ th bit of $a_{i}$ is 1 and the $l$ th bit of $b_{j}$ is 0 , then $\left(u_{i}, w_{l}, v_{j}\right)$ is a length-2 path between $s$ and $t$.

- Consider the case $a_{i}=b_{j}$. We show that $\operatorname{dist}(s, t)=3$. Note that there is no index $l \in[\ell]$ such that $a_{i}$ and $b_{j}$ differ at the $l$ th bit. Thus, each $w_{l} \in V_{C}$ and $x_{l} \in V_{D}$ is adjacent to exactly one of $\left\{u_{i}, v_{j}\right\}$. Hence there is no length-2 path between $s$ and $t$.

Therefore, if $S_{A} \cap S_{B}=\emptyset$, then $\operatorname{dist}(s, t)=2$ for all pairs $\{s, t\}$, and so the diameter is 2; otherwise, there exist $s=u_{i} \in V_{A}$ and $t=v_{j} \in V_{B}$ such that $\operatorname{dist}(s, t)=3$, and so the diameter is 3 .

Reduction. Suppose that there is a randomized distributed algorithm $\mathcal{A}$ that is able to compute the diameter with $o\left(n / \log ^{2} n\right)$ energy per vertex, with failure probability $f=1 / \operatorname{poly}(n)$. We show that the algorithm $\mathcal{A}$ can be transformed into a randomized communication protocol that solves the set-disjointness problem with $o(n)$ bits of communication, and with the same failure probability $f=1 / \operatorname{poly}(n)$.

The main challenge in the reduction is that we do not impose any message size constraint. To deal with this issue, our strategy is to consider a modified computation model $\mathcal{M}^{\prime}$. We will endow the vertices in the modified computation model $\mathcal{M}^{\prime}$ with strictly more capabilities than the original radio network. Then, we argue that in the setting of $\mathcal{M}^{\prime}$, we can assume that each message has size $O(\log k)$.

Modified Computation Model $\mathcal{M}^{\prime}$. We add the following extra powers to the vertices:

(P1) All vertices have access to an infinite shared random string. They know the vertex set and the IDs of all vertices. Specifically, $\operatorname{ID}\left(w_{i}\right)=i$ for each $w_{i} \in V_{C} ; \operatorname{ID}\left(x_{i}\right)=\ell+i$ for each $x_{i} \in V_{D} ; \operatorname{ID}\left(u^{\star}\right)=2 \ell+1 ; \operatorname{ID}\left(v^{\star}\right)=2 \ell+2$. Thus, for each $v \in V_{C} \cup V_{D} \cup\left\{u^{\star}, v^{\star}\right\}$, its role can be inferred from $\operatorname{ID}(v)$.

(P2) Messages received by vertices in $V_{C} \cup V_{D} \cup\left\{u^{\star}, v^{\star}\right\}$ (according to the usual radio network rules) are immediately communicated to all vertices. For example, if $v \in V_{C} \cup V_{D} \cup\left\{u^{\star}, v^{\star}\right\}$ receives $m$ from $u \in V$ at time $t$, then by the end of round $t$ all vertices in $V$ know that " $v$ receives $m$ from $u$ at time $t . "$ 
(P3) Each vertex $v \in V_{A} \cup V_{B}$ knows the list of the IDs of its neighbors initially.

Next, we discuss the consequences of these extra powers. In particular, we show that we can make the following assumptions about algorithms in this modified model $\mathcal{M}^{\prime}$.

Vertices in $V_{C} \cup V_{D} \cup\left\{u^{\star}, v^{\star}\right\}$ Never Transmit. Powers (P1) and (P2) together imply that each vertex in the graph is able to locally simulate the actions of all vertices in $V_{C} \cup V_{D} \cup\left\{u^{\star}, v^{\star}\right\}$. Intuitively, this means that all vertices in $V_{C} \cup V_{D} \cup\left\{u^{\star}, v^{\star}\right\}$ do not need to transmit at all throughout the algorithm.

Note that each vertex $v \in V$ already knows the list of $N(v) \cap\left(V_{C} \cup V_{D} \cup\left\{u^{\star}, v^{\star}\right\}\right)$. If $v \in V_{A} \cup V_{B}$, then $v$ knows this information via (P3). If $v \in V_{C} \cup V_{D} \cup\left\{u^{\star}, v^{\star}\right\}$, then $v$ knows this information via (P1); the role of each vertex in $V_{C} \cup V_{D} \cup\left\{u^{\star}, v^{\star}\right\}$ can be inferred from its ID, which is a public to everyone.

Thus, right before the beginning of each time $t$, each vertex $v \in V$ already knows exactly which vertices in $N(v) \cap\left(V_{C} \cup V_{D} \cup\left\{u^{\star}, v^{\star}\right\}\right)$ will transmit at time $t$ and their messages. Thus, in the modified model $\mathcal{M}^{\prime}$, we can simulate the execution of an algorithm which allows the vertices in $V_{C} \cup V_{D} \cup\left\{u^{\star}, v^{\star}\right\}$ to transmit by another algorithm that forbid them to do so.

Messages Sent by Vertices in $V_{A} \cup V_{B}$ Have Length $O(\log k)$. Next, we argue that we can assume that each message $m$ sent by a vertex $v^{\prime} \in V_{A} \cup V_{B}$ can be replaced by another message $m^{\prime}$ which contains only the list of all neighbors of $v^{\prime}$, and this can be encoded as an $O(\log k)$-bit message, as follows. Recall that $N\left(v^{\prime}\right)$ is a subset of $V_{C} \cup V_{D} \cup\left\{u^{\star}, v^{\star}\right\}$, and so we can encode $N\left(v^{\prime}\right)$ as a binary string of length $\left|V_{C} \cup V_{D} \cup\left\{u^{\star}, v^{\star}\right\}\right|=2 \ell+2=O(\log k)$.

The message $m$ is a function of all information that $v^{\prime}$ has. Since no vertex in $V_{C} \cup V_{D} \cup\left\{u^{\star}, v^{\star}\right\}$ transmits any message, $v^{\prime}$ never receives a message, and so the information that $v^{\prime}$ has consists of only the following components.

- The shared randomness and the ID list of all vertices (due to (P1)).

- The history of vertices in $V_{C} \cup V_{D} \cup\left\{u^{\star}, v^{\star}\right\}$ (due to (P2)).

- The list of neighbors of $v^{\prime}$ (due to (P3)).

The only private information that $v^{\prime}$ has is its list of neighbors. If a vertex $u^{\prime} \in V$ knows the list of neighbors of $v^{\prime}$, then $u^{\prime}$ is able to calculate $m$ locally, and so $v^{\prime}$ can just send its list of neighbors in lieu of $m$.

Algorithm $\mathcal{A}^{\prime}$. To sum up, given the algorithm $\mathcal{A}$, we can transform it into another algorithm $\mathcal{A}^{\prime}$ in the modified computation model $\mathcal{M}^{\prime}$ that uses only $O(\log k)$-bit messages, and $\mathcal{A}^{\prime}$ achieves what $\mathcal{A}$ does. Note that the energy cost of $\mathcal{A}^{\prime}$ is at most the energy cost $\mathcal{A}$.

Solving Set-Disjointness. Now we show how to transform $\mathcal{A}^{\prime}$ into a protocol for the setdisjointness problem using only $o(k)$ bits of communication. The protocol is simply a simulation of $\mathcal{A}^{\prime}$. The shared random string used by $\mathcal{A}^{\prime}$ is the same random string shared by the two players $A$ and $B$.

Each player $X \in\{A, B\}$ is responsible for simulating vertices in $V_{X}$. Vertices in $V_{A}$ and $V_{B}$ never receive messages, and so all we need to do is let both players $A$ and $B$ know the messages sent to $V_{C} \cup V_{D} \cup\left\{u^{\star}, v^{\star}\right\}$ (in view of $(\mathrm{P} 2)$ ). 
We show how to simulate one round $\tau$ of $\mathcal{A}^{\prime}$. Let $Z(\tau)$ be the subset of vertices in $V_{C} \cup V_{D} \cup$ $\left\{u^{\star}, v^{\star}\right\}$ that listen at time $\tau$, and consider a vertex $u^{\prime} \in Z(\tau)$. (Recall that everyone can predict the action of every vertex in $V_{C} \cup V_{D} \cup\left\{u^{\star}, v^{\star}\right\}$.)

Let $Q_{A}$ be the number of vertices in $N\left(u^{\prime}\right) \cap V_{A}$ transmitting at time $\tau$. We define $m_{u^{\prime}, \tau, A}$ as follows.

$$
m_{u^{\prime}, \tau, A}= \begin{cases}\text { " } 0 " & \text { if } Q_{A}=0 . \\ " \geq 2 " & \text { if } Q_{A} \geq 2 . \\ \left(v^{\prime}, m^{\prime}\right) & \text { if } Q_{A}=1, \text { and } v^{\prime} \text { is the vertex in } N\left(u^{\prime}\right) \cap V_{A} \text { sending } m^{\prime} \text { at time } \tau .\end{cases}
$$

We define $m_{u^{\prime}, \tau, B}$ analogously. Note that the length of $m^{\prime}$ must be $O(\log k)$ bits.

The protocol for simulating round $\tau$ is simply that $A$ sends $m_{u^{\prime}, \tau, A}$ (for each $u^{\prime} \in Z(\tau)$ ) to $B$, and $B$ sends $m_{u^{\prime}, \tau, B}$ (for each $u^{\prime} \in Z(\tau)$ ) to $A$. This offers enough information for both player to know the channel feedback (noise, silence, or a message $m$ ) received by each vertex in $Z(\tau)$. Note that the number of bits exchanged by $A$ and $B$ due to the simulation of round $\tau$ is $O(|Z(\tau)| \log k)$.

Recall that the energy cost of each vertex in an execution of $\mathcal{A}^{\prime}$ is $o\left(k / \log ^{2} k\right)$, and we have $\left|V_{C} \cup V_{D} \cup\left\{u^{\star}, v^{\star}\right\}\right|=O(\log k)$. Thus, the total number of bits exchanged by the two players $A$ and $B$ is

$$
\sum_{\tau} O(|Z(\tau)| \log k)=\left|V_{C} \cup V_{D} \cup\left\{u^{\star}, v^{\star}\right\}\right| \cdot o\left(k / \log ^{2} k\right) \cdot O(\log k)=o(k) .
$$

We remark that the proof of Theorem 5.2 can be extended to graphs with higher diameter by using a slightly more complicated lower bound graph construction and analysis; see e.g., 9 . Intuitively, this is due to the fact that the lower bound graph is sparse, so we are able to subdivide the edges.

\subsection{Upper Bounds}

The approximation ratios in Theorems 5.1 and 5.2 cannot be improved. Observe that BFS already gives a 2-approximation of diameter, as $D^{\prime}=\max _{u \in V(G)}\left\{\operatorname{dist}_{G}(s, u)\right\} \in[\operatorname{diam}(G) / 2, \operatorname{diam}(G)]$, and we know that a BFS can be computed in $n^{o(1)}$ energy.

If we allow an energy budget of $n^{\frac{1}{2}+o(1)}$ then it is possible to achieve a nearly $3 / 2$-approximation by applying the algorithm of [19, 38, which computes a $D^{\prime}$ such that $\lfloor 2 \operatorname{diam}(G) / 3\rfloor \leq D^{\prime} \leq \operatorname{diam}(G)$. More precisely, if we write $\operatorname{diam}(G)=3 h+z$, where $h$ is a non-negative integer, and $z \in\{0,1,2\}$, then $D^{\prime} \in[2 h+z, \operatorname{diam}(G)]$ for the case $z=0,1$, and $D^{\prime} \in[2 h+1, \operatorname{diam}(G)]$ for the case $z=2$. Note that this does not contradict the $\Omega(n)$ energy lower bound for distinguishing between $\operatorname{diam}(G)=1$ and $\operatorname{diam}(G)=2$ in Theorem 5.1, nor does it contradict Theorem 5.2.

The algorithm of [19, 38] is as follows. Let each vertex join $S$ with probability $(\log n) / \sqrt{n}$, and compute a BFS from each vertex in $S$. Let $v^{\star}$ be any vertex that maximizes the distance to $S$. Identify any set of $\sqrt{n}$ vertices $R$ that are the closest to $v^{\star}$, and compute a BFS from each vertex in $R$. The diameter approximation $D^{\prime}$ is the maximum BFS-label computed throughout the algorithm. Note that there are multiple valid choice of $v^{\star}$ and $R$, and the tie can be broken arbitrarily 8 Since BFS can be computed in $n^{o(1)}$ energy, with a suitable implementation, this algorithm be executed using $n^{\frac{1}{2}+o(1)}$ energy. For the sake of completeness, in what follows we provide the detail for an implementation, which is based on the following subroutines.

\footnotetext{
${ }^{8}$ Precisely, it is required that $|R|=\sqrt{n}$, and for each $u \in R$, there are less than $\sqrt{n}$ vertices $v$ such that dist $\left(v, v^{\star}\right)<$ $\operatorname{dist}\left(u, v^{\star}\right)$. In general, there could be multiple choices of $R$ satisfying this requirement.
} 
Leader Election: Elect a leader $v_{0} \in V$ such that all vertices know $\operatorname{ID}\left(v_{0}\right)$. It is known that this task can be solved in $\tilde{O}(n)$ time and $\tilde{O}(1)$ energy [10].

Find Minimum: Suppose there is already a leader $v_{0} \in V$, and each vertex $u \in V$ knows $\operatorname{dist}\left(u, v^{\star}\right)$. Each vertex $u$ holds an integer $k_{u} \in[1, K]$ and a message $m_{u}$. The goal is to elect one vertex $u^{\star}$ such that $k_{u^{\star}}=\min \left\{k_{u} \mid u \in V\right\}$ and have all vertices know $m_{u^{\star}}$. Tie is broken arbitrarily. The task Find Maximum is defined analogously.

We argue that the task Find Minimum and Find Maximum can be solved in $\tilde{O}(\operatorname{diam}(G))$ time and $\tilde{O}(1)$ energy, given that $K=O(\operatorname{poly}(n))$. To solve this task, we will do a binary search. Let $I \subseteq[1, K]$ be an interval currently under consideration. We let $v_{0}$ test whether there exists a vertex $u^{\prime}$ with $k_{u^{\prime}} \in I$ by doing $O(\operatorname{diam}(G))$ Local-Broadcasts on the BFS tree, layer by layer. The root $v_{0}$ is able to announce the result to everyone, also using $O(\operatorname{diam}(G))$ Local-Broadcasts on the BFS tree, layer by layer. After $O(\log K)=\tilde{O}(1)$ iterations, we are done.

Theorem 5.3. There is an algorithm that computes a 2-approximation of diameter in $n^{1+o(1)}$ time and $n^{o(1)}$ energy.

Proof. Apply Leader Election to elect a leader $v_{0}$, do a BFS from $v_{0}$, and then do a Find Maximum to let each vertex learn $\max \left\{\operatorname{dist}\left(u, v_{0}\right) \mid u \in V\right\}$. This gives a 2-approximation of the diameter $D$.

Theorem 5.4. There is an algorithm that computes an approximation $D^{\prime}$ such that $\lfloor 2 \operatorname{diam}(G) / 3\rfloor \leq$ $D^{\prime} \leq \operatorname{diam}(G)$ in $n^{3 / 2+o(1)}$ time and $n^{1 / 2+o(1)}$ energy.

Proof. We show how to implement the algorithm of [19, 38]. We first apply Leader Election to elect a leader $v_{0}$, and do a BFS from $v_{0}$, we will use this tree to do Find Minimum and Find Maximum in subsequent steps of the algorithm.

In the algorithm of [19, 38], we let each vertex join $S$ with probability $(\log n) / \sqrt{n}$. Using $|S|=\tilde{O}(\sqrt{n})$ iterations of Find Minimum we can let everyone know the IDs of vertices in $S$. Then, we sequentially compute a BFS from each vertex in $S$. Let $v^{\star}$ be a vertex that maximizes the distance to $S$. Such a vertex $v^{\star}$ can be elected using one iteration of Find Maximum. To compute the set $R$, we first do a BFS from $v^{\star}$ so that everyone knows its distance to $v^{\star}$. Then, after $\sqrt{n}$ iterations of Find Minimum, we can let everyone learn the set $R$, and then we can do the BFS computation from each vertex in $R$ sequentially. The diameter approximation $D^{\prime}$ is the maximum BFS-label computed throughout the algorithm, and this can be computed using one iteration of Find Maximum. It is clear that the algorithm takes $n^{3 / 2+o(1)}$ time and $n^{1 / 2+o(1)}$ energy, as it only uses $\tilde{O}(\sqrt{n})$ Find Minimum, Find Maximum, and BFS computations. 


\section{References}

[1] A. Abboud, K. Censor-Hillel, and S. Khoury. Near-linear lower bounds for distributed distance computations, even in sparse networks. In C. Gavoille and D. Ilcinkas, editors, Distributed Computing (DISC), pages 29-42. Springer Berlin Heidelberg, 2016.

[2] N. Alon, A. Bar-Noy, N. Linial, and D. Peleg. A lower bound for radio broadcast. Journal of Computer and System Sciences, 43(2):290-298, 1991.

[3] R. Bar-Yehuda, O. Goldreich, and A. Itai. Efficient emulation of single-hop radio network with collision detection on multi-hop radio network with no collision detection. Distributed Computing, 5(2):67-71, 1991.

[4] R. Bar-Yehuda, O. Goldreich, and A. Itai. On the time-complexity of broadcast in multihop radio networks: An exponential gap between determinism and randomization. Journal of Computer and System Sciences, 45(1):104-126, 1992.

[5] M. Barnes, C. Conway, J. Mathews, and D. K. Arvind. ENS: An energy harvesting wireless sensor network platform. In Proceedings of the 5th International Conference on Systems and Networks Communications (ICSNC), pages 83-87, 2010.

[6] M. Bender, T. Kopelowitz, S. Pettie, and M. Young. Contention resolution with constant throughput and log-logstar channel accesses. SIAM J. Comput., 47:1735-1754, 2018.

[7] P. Berenbrink, C. Cooper, and Z. Hu. Energy efficient randomised communication in unknown adhoc networks. Theoretical Computer Science, 410(27):2549 - 2561, 2009.

[8] M. Braverman and A. Moitra. An information complexity approach to extended formulations. In Proceedings of the 45th Annual ACM Symposium on Theory of Computing (STOC), pages 161-170, New York, NY, USA, 2013. ACM.

[9] K. Bringmann and S. Krinninger. A note on hardness of diameter approximation. Information Processing Letters, 133:10-15, 2018.

[10] Y.-J. Chang, V. Dani, T. P. Hayes, Q. He, W. Li, and S. Pettie. The energy complexity of broadcast. In Proceedings of the 2018 ACM Symposium on Principles of Distributed Computing (PODC), pages 95-104, 2018.

[11] Y.-J. Chang, T. Kopelowitz, S. Pettie, R. Wang, and W. Zhan. Exponential separations in the energy complexity of leader election. In Proceedings of the 49th Annual ACM SIGACT Symposium on Theory of Computing (STOC), pages 771-783, 2017.

[12] I. Chlamtac and S. Kutten. On broadcasting in radio networks-problem analysis and protocol design. IEEE Transactions on Communications, 33(12):1240-1246, 1985.

[13] I. Chlamtac and S. Kutten. Tree-based broadcasting in multihop radio networks. IEEE Trans. Computers, 36(10):1209-1223, 1987.

[14] A. Czumaj and P. Davies. Exploiting spontaneous transmissions for broadcasting and leader election in radio networks. In Proceedings of the 2017 ACM Symposium on Principles of Distributed Computing (PODC), pages 3-12, 2017. 
[15] L. Gasieniec, E. Kantor, D. R. Kowalski, D. Peleg, and C. Su. Energy and time efficient broadcasting in known topology radio networks. In Proceedings 21st International Symposium on Distributed Computing (DISC), pages 253-267, 2007.

[16] M. Ghaffari and B. Haeupler. Near-optimal BFS-tree construction in radio networks. IEEE Communications Letters, 20(6):1172-1174, 2016.

[17] S. Gilbert, V. King, S. Pettie, E. Porat, J. Saia, and M. Young. (Near) optimal resourcecompetitive broadcast with jamming. In Proceedings of the 26th ACM Symposium on Parallelism in Algorithms and Architectures (SPAA), pages 257-266, 2014.

[18] B. Haeupler and D. Wajc. A faster distributed radio broadcast primitive. In Proceedings 35th ACM Symposium on Principles of Distributed Computing (PODC), pages 361-370. ACM, 2016.

[19] S. Holzer, D. Peleg, L. Roditty, and R. Wattenhofer. Brief announcement: Distributed 3/2approximation of the diameter. In Proc. 28th International Symposium on Distributed Computing (DISC 2014), pages 562-564. Springer, 2014.

[20] T. Jurdzinski, M. Kutylowski, and J. Zatopianski. Efficient algorithms for leader election in radio networks. In Proceedings of the 21st Annual ACM Symposium on Principles of Distributed Computing (PODC), pages 51-57, 2002.

[21] T. Jurdzinski, M. Kutylowski, and J. Zatopianski. Energy-efficient size approximation of radio networks with no collision detection. In Proceedings of the 8th Annual International Conference on Computing and Combinatorics (COCOON), pages 279-289, 2002.

[22] T. Jurdzinski, M. Kutylowski, and J. Zatopianski. Weak communication in radio networks. In Proceedings of the 8th International European Conference on Parallel Computing (Euro-Par), pages 965-972, 2002.

[23] T. Jurdzinski, M. Kutylowski, and J. Zatopianski. Weak communication in single-hop radio networks: adjusting algorithms to industrial standards. Concurrency and Computation: Practice and Experience, 15(11-12):1117-1131, 2003.

[24] T. Jurdzinski and G. Stachowiak. Probabilistic algorithms for the wakeup problem in singlehop radio networks. In Proceedings of the 13th International Symposium on Algorithms and Computation (ISAAC), pages 535-549, 2002.

[25] J. Kabarowski, M. Kutylowski, and W. Rutkowski. Adversary immune size approximation of single-hop radio networks. In Proceedings Third International Conference on Theory and Applications of Models of Computation (TAMC), pages 148-158, 2006.

[26] B. Kalyanasundaram and G. Schnitger. The probabilistic communication complexity of set intersection. SIAM J. Discrete Math., 5(4):545-557, 1992.

[27] M. Kardas, M. Klonowski, and D. Pajak. Energy-efficient leader election protocols for singlehop radio networks. In Proceedings 42nd International Conference on Parallel Processing (ICPP), pages 399-408, 2013.

[28] V. King, S. Pettie, J. Saia, and M. Young. A resource-competitive jamming defense. Distributed Computing, 31:419-439, 2018. 
[29] M. Klonowski and D. Pajak. Brief announcement: Broadcast in radio networks, time vs. energy tradeoffs. In Proceedings 37th ACM Symposium on Principles of Distributed Computing (PODC), pages 115-117, 2018.

[30] M. Klonowski and M. Sulkowska. Energy-optimal algorithms for computing aggregative functions in random networks. Discrete Mathematics $\&$ Theoretical Computer Science, 17(3):285306, 2016.

[31] E. Kushilevitz and Y. Mansour. An $\Omega(D \log (N / D))$ lower bound for broadcast in radio networks. SIAM Journal on Computing, 27(3):702-712, 1998.

[32] M. Kutylowski and W. Rutkowski. Adversary immune leader election in ad hoc radio networks. In Proceedings 11th Annual European Symposium on Algorithms (ESA), pages 397-408, 2003.

[33] G. L. Miller, R. Peng, A. Vladu, and S. C. Xu. Improved parallel algorithms for spanners and hopsets. In Proceedings of the 27th ACM on Symposium on Parallelism in Algorithms and Architectures (SPAA), pages 192-201, 2015.

[34] G. L. Miller, R. Peng, and S. C. Xu. Parallel graph decompositions using random shifts. In Proceedings of the 25th Annual ACM Symposium on Parallelism in Algorithms and Architectures (SPAA), pages 196-203, 2013.

[35] K. Nakano and S. Olariu. Energy-efficient initialization protocols for single-hop radio networks with no collision detection. IEEE Trans. Parallel Distrib. Syst., 11(8):851-863, 2000.

[36] C. Newport. Radio network lower bounds made easy. In Proceedings of the 28th International Symposium on Distributed Computing (DISC), pages 258-272, 2014.

[37] J. Polastre, R. Szewczyk, and D. Culler. Telos: enabling ultra-low power wireless research. In Proceedings of the 4th International Symposium on Information Processing in Sensor Networks (IPSN), pages 364-369, 2005.

[38] L. Roditty and V. V. Williams. Fast approximation algorithms for the diameter and radius of sparse graphs. In Proceedings 45th ACM Symposium on Theory of Computing (STOC), pages $515-524,2013$. 